\title{
Computational study of the sensitivity of laser light scattering particle sizing to refractive index and irregularity.
}

Juan Carlos Gómez Martín ${ }^{1 *}$, Daniel Guiradoํㅡ, Evgenij Zubko², Jesús Escobar-Cerezo ${ }^{1}$, Fernando Moreno $^{1}$, Olga Muñoz ${ }^{1}$

${ }^{1}$ Instituto de Astrofísica de Andalucía - CSIC, 18008, Granada, Spain

${ }^{2}$ Kyung Hee University, Humanitas College, Yongin-shi, Kyungki-do 17104, Korea

*Email:jcgomez@iaa.es

This work is licensed under a CC-BY-NC-ND

\begin{abstract}
Measuring the size distribution of dust particles is of interest in many scientific and technological contexts. One of the most widely used techniques is laser light scattering (LLS), which provides the distribution of surface-equivalent spheres that fits the observed angular dependence of light scattered by a sample. We have revisited the problem of the uncertain lower size limit of this method by simulating laboratory measurements of the light intensity scattered by polydisperse spheres and irregular particles (agglomerated debris and pocked spheres), from which the original distributions are retrieved by regularized inversion with Mie and Fraunhofer phase functions. For the usual combination of blue $(\lambda=466 \mathrm{~nm})$ and red $(\lambda=633 \mathrm{~nm})$ light sources, size distributions of spheres with radii $r>0.1 \mu \mathrm{m}$ are retrieved with Mie if the true complex refractive index $(m=n-i k)$ is used. The retrieval for $0.1 \mu \mathrm{m}<r<3 \mu \mathrm{m}$ is sensitive to errors in the assumed $m$, which results primarily from the dependence of the scattering efficiency $Q_{\text {sca }}$ on $m$. Irregular particle shape has also an impact on the $Q_{\text {sca }} v s$. $r$ curves, whose maxima are shifted towards larger $r$ and are smoother compared to spherical particles. For a violet-blue wavelength $(\lambda=442 \mathrm{~nm})$, good retrievals are obtained for irregular particles with $r>1 \mu \mathrm{m}$ even if $m$ is not very well known or the Fraunhofer model is used. Spurious slumps and enhancements appear for $r<1 \mu \mathrm{m}$, although if $n$ is known, the actual lower limit decreases for increasing $n$. This implies that LLS size distributions of submicron irregular particles may not be accurate. Establishing the lower size limit requires inspection of the dependence on $m$ and analysis of the irregularity of samples.
\end{abstract}

\section{Keywords}

Particle Sizing, Size Distribution, Laser Light Scattering, Irregular Particles 


\section{Introduction}

The physical properties of atmospheric aerosols, of cometary and interplanetary dust and of particulate deposits on the surface of airless bodies determine the way in which they scatter light as a function of illumination and viewing angles, and wavelength. The analysis of remote and close-range observations of the intensity and degree of linear polarization of sunlight scattered by particle clouds and particlecovered surfaces in atmospheric and astronomical environments, with the assistance of modelling and laboratory data, provides a powerful method for retrieving their properties and discussing their implications in a wider context [1]. Remote atmospheric and astronomical observations of dust clouds are usually interpreted using models of the interaction between matter and radiation [2-8] or by direct comparison with laboratory measurements of the scattering matrix of appropriated dust analogs [9-11].

Additionally, the models are tested and validated by analysis of laboratory measurements [12-16].

The effect of particle size, shape, internal structure and composition are heavily entangled in light scattering data. Laboratory dust analogs obtained from milled and sieved mineral and meteoritic samples or synthesised using bottom-up methods present generally broad size distributions, which results in sizeaveraging of their scattering properties. Thus, separating the effect of size in laboratory observations is a key first step towards the retrieval of other properties such as shape and refractive index. A common approach to address this problem is determining the size distribution of samples using particle sizing techniques before performing more complex spectro-photopolarimetric measurements $[17,18]$.

Among the particle sizing methods, low-angle laser light scattering (LLS) [19] has become one of the most widely used techniques in many different scientific and technological fields, owing to its measurement speed, bulk-representative sampling, suitability for a wide range of sizes and materials and ease of use [20]. Natural dust particles are generally irregularly shaped, which makes necessary adopting a convention on what size means. Bulk-representative sampling methods consider an equivalent distribution of spheres, with size meaning the radius of spheres equivalent in some physical sense to the particles under study (e.g. aerodynamic radius in the case of sedimentation and radius of a projected surface-equivalent sphere in the case of LLS). Thus, the distributions obtained from sedimentation, Coulter counting and LLS have similar shape but generally different absolute values [2124]. LLS provides a size distribution of a natural dust sample represented by the distribution of projected surface-equivalent spheres that fits the observed angular distribution of the intensity of light scattered by the sample. Using spheres as model particles is convenient because Mie theory provides exact results for their phase function and it does not require vast computational resources. LLS relies on the fact that diffraction angle is inversely related to particle size. At low scattering angles (forward scattering), the most important factor determining the scattering behaviour of particles is size, while shape, structure and refractive index have minor or negligible influence on the retrieved size distribution. The forward scattering direction is mostly sensitive to larger particles. By contrast, the scattered intensity for scattering angle larger than $\sim 10^{\circ}$ is sensitive to the concentration of smaller particles in the size 
distribution as well as shape, porosity and refractive index. Large scattering angles are usually not considered to avoid sphere-specific features (deep minimum at $100^{\circ}-120^{\circ}$ and rainbows [7, 25]). Nonsphericity of target particles is a potential pitfall of the LLS technique in the retrieval of size distributions in the small particle size range. The reliability of LLS and the validity of its assumptions regarding the overlapping effects of shape, size and composition have been a matter of concern for many years, not least because of the obscurity of the analysis methods implemented by commercial sizers.

The aim of this study is delimiting the range of validity of the LLS sizing technique for spherical particles and for irregular particles with unit average aspect ratio (i.e., equi-dimensional) in the critical range where particle size and wavelength are comparable (i.e. within a factor of $\sim 10$ ). This range has been shown to play an important role [26], for example, on the appearance of a negative branch in the linear polarization phase curve $[27,28]$, which is one of the distinct features of polarimetric observations of cometary dust tails. To this end, we have forward-modelled the angular distribution of the intensity of light scattered by polydisperse spheres and model irregular particles, and these have been analysed with the LLS particle sizing method in order to retrieve the underlying size distribution and discuss the entanglement of size, refractive index and irregularity effects on the retrievals based on the quality of the fit and the correspondence with the original distribution.

This paper is organized as follows. Section 2 summarizes the main theoretical aspects of light scattering by dust particles and of regularized inversion. Section 3 describes the computational techniques used to model and analyse the phase function of particle clouds. Section 4.1 presents the application of the LLS method to spheres and the propagation of error in the refractive index to the retrieved size distribution. Section 4.2 deals with the effect of irregularity in the sizing of small particles using spheres as the reference regular geometry. Results are discussed in Section 5. Finally, Section 6 summarizes the findings of this work and concludes with some remarks about the interpretation of LLS data.

\section{Theory}

\subsection{The scattering matrix}

A beam of monochromatic light is described by its Stokes vector. The interaction between a light beam with Stokes vector $\boldsymbol{\Phi}_{0}$ and a cloud of particles results in scattering of the incident light and modification of the Stokes parameters. The Stokes vector of the scattered beam $\boldsymbol{\Phi}$ is related to $\boldsymbol{\Phi}_{0}$ by the scattering matrix $\mathbf{F}$ :

$\boldsymbol{\Phi}=\frac{\lambda^{2}}{4 \pi^{2} D^{2}} \mathbf{F} \boldsymbol{\Phi}_{0}$

where $\lambda$ is the wavelength of the incident beam and $D$ is the distance between the particle cloud and the position at which the Stokes components are measured. The components of $\mathbf{F}$ depend on the physical properties of the particles (number density, size, shape, internal structure and composition), as well as on geometrical parameters (particle orientation and scattering direction). When the particles are 
randomly oriented all scattering planes are equivalent, and the scattering direction is fully described by the scattering angle $(\theta)$, i.e. the angle between the propagation directions of the incident and scattered beams. Furthermore, if the ensemble of particles presents mirror symmetry, only six non-zero unique matrix elements remain $[29,30]$. The normalized scattering matrix is given by:

$\mathbf{F}=\frac{\sigma_{\text {sca }}}{4 \pi} \mathbf{P}=\frac{r^{2} Q_{\text {sca }}}{4} \mathbf{P}$

where $\sigma_{\mathrm{sca}}$ is the average scattering cross section and $Q_{\mathrm{sca}}=\sigma_{\mathrm{sca}} /\left(\pi r^{2}\right)$ the scattering efficiency [31]. For unpolarized incident light and assuming that a low sample density grants the assumption of single scattering, the first element of the scattering matrix, $F_{11}(\theta, \lambda)$, known as the phase function, is proportional to the intensity $I(\theta, \lambda)$, which represents the probability of scattering in any given direction. $I(\theta, \lambda)$, under the aforementioned conditions, is a relative-scale measurement of the phase function. Hereafter we refer to $I(\theta, \lambda)$ as the (measured or forward-modelled) average phase function. The LLS technique relies on measuring of $I(\theta, \lambda)$ to determine particle size.

\subsection{The size distribution}

The phase function of a polydisperse particulate sample is an average of the phase functions of individual sizes weighted by the size distribution of the sample:

$$
I(\theta, \lambda) \propto P_{11}(\theta, \lambda)=\int_{r_{1}}^{r_{2}} \sigma_{\mathrm{sca}}(x) P_{11}(x, \theta) n(r) d r
$$

Since for irregular particles size is not well defined, the measured phase function $I(\theta, \lambda)$ is interpreted as the average of the phase functions of equivalent spheres weighted by their size distribution, where size is understood as the radius of the sphere with the same geometric cross section. In Eq. (3), $P_{11}$ is the first element of the normalized scattering matrix (from Eq. (2)), $x=2 \pi r / \lambda$ is the size parameter and $n(r)=d \bar{N}(r) / \mathrm{d} r$ is the number density size distribution. $\bar{N}(r)$ is the cumulative size distribution and $n(r) d r$ is the number of particles with size between $r$ and $r+d r$. A size distribution $n(r)$ is usually described by its effective radius $r_{\text {eff }}$ and effective standard deviation $\sigma_{\text {eff }}$ as defined by Hansen and Travis [25].

It is advantageous to introduce the $r \rightarrow \log r$ variable transformation in Eq. (3) and use the size distribution in logarithmic scale, for two reasons:

(i) when plotting $d \bar{N}(r) / d \log r=\ln 10 r n(r) v s . \log r$, equal areas under parts of the curve means equal relative amounts of spheres in the ranges considered.

(ii) $\quad P_{11}$ shows smoother variability for equal relative steps $\Delta r / r$ (i.e., for equal logarithmic steps) than for equal absolute steps $\Delta r$ [32].

Different kinds of size distributions $N_{i}(\log r) \equiv d \bar{N}_{i}(r) / d \log r=\ln 10 r n_{\mathrm{i}}(r)$ can be considered. The index $i$ denotes the type of distribution: number $(i=0$, denoted alternatively as $N(\log r)$ ), area $(i=2$, denoted alternatively as $S(\log r)=d \bar{S}(r) / d \log r=\ln 10 r s(r))$ and volume $(i=3$, denoted alternatively as $V(\log r)=d \bar{V}(r) / d \log r=\ln 10 r v(r))$. With these changes, Eq. (3) can be re-written as: 


$$
I(\theta, \lambda)=C \int_{r_{1}}^{r_{2}} \frac{\sigma_{\mathrm{sca}}(x) P_{11}(x, \theta)}{g_{i}(r)} N_{i}(\log r) d \log r
$$

where $g_{i}(r)=r^{i}$ [32]. The usual choice of $i=2$, i.e. $N_{2}(\log r)=S(\log r) \propto v(r)$, is of a practical nature and bears no influence in the fact that it is $\sigma_{\text {sca }}$ (with units of area) what defines what size means in Eq. (4), i.e. the radius of the projected area [33]. The reason for using the logarithmic scale projected area or equivalently the volume distribution is that it weights particles by $r^{-3}$, which allows reducing the huge dynamic range of the scattering intensity pattern [19]. Commercial sizers report the retrieved distribution as a volume-based histogram where the value for each size bin represents the fraction of the total particle volume that correspond to particles with equivalent radii inside that size bin. Note that this is the volume of the sphere with equivalent projected area, not with equivalent volume. The intensity measured by any given detector $j$ with efficiency $\phi_{j}$ of a particle sizer is an integration of the phase function over the solid angle subtended by the detector's surface [34]. Therefore, Eq. (4) may be re-written as:

$$
I\left(\theta_{j}, \lambda\right)=\int_{\Omega_{j}} I(\theta, \lambda) d \Omega=C \int_{r_{1}}^{r_{2}} \frac{\sigma_{\mathrm{sca}}(x)}{r^{2}}\left\{\phi_{\mathrm{j}} \int_{\Omega_{j}} P_{11}(\theta, x) d \Omega\right\} S(\log r) d \log r
$$

Eq. (5) can be discretized as follows [34]:

$$
\mathbf{b}=\mathbf{K} \mathbf{s}
$$

In Eq. (6), b is the phase function vector with $N_{\theta}$ rows (number of scattering angle bins), where each element is the average of the phase function within a narrow scattering angle bin. $\mathbf{K}$ is a matrix where each column $i$ contains the product of the average phase function of particles within a specific size bin $i$ for $N_{\theta}$ angular detectors, $P_{11}\left(x_{i}, \theta_{j}\right)$ weighted by $r^{-2}$, and the logarithmic width of the bin:

$\mathrm{K}_{i j}=Q_{\text {scat }}\left(r_{i}\right) P_{11}\left(x_{i}, \theta_{j}\right) \Delta \log r_{i}$

The matrix $\mathbf{K}$ has $N_{\theta}$ rows (number of angular bins) and $N_{r}$ columns (number of size bins). The columns of this matrix are usually obtained from Fraunhofer diffraction or from Mie theory. The column vector $\mathbf{s}\left(N_{r}\right.$ rows) contains the discretized logarithmic scale distribution $\left(s_{i}=S\left(\log r_{i}\right)\right)$.

The 'observational' vector $\mathbf{b}$ can be the phase function measured by a particle sizer, or a synthetic phase function obtained by direct application of Eq. (6) (i.e. forward-modelling). Forward modelling is carried out by filling the columns of $\mathbf{K}$ with phase functions generated with a model of the interaction between radiation and dust particles, and the elements of $\mathbf{s}$ with the chosen size distribution. Given an observed or a forward-modelled vector b, Eq. (6) can also be inverted to retrieve $\mathbf{s}$ :

$\mathbf{s}=\mathbf{D} \mathbf{b}$

where $\mathbf{D}$ is the generalized inverse of $\mathbf{K}$. Particle sizers estimate the unknown vector $\mathbf{s}$ by using Eq. (8).

\subsection{The inversion method}

An inversion such as Eq. (8) can be performed in principle by solving the least-squares problem:

$$
\mathbf{s}_{\mathrm{LSQ}}=\arg \min \|\mathbf{K} \mathbf{s}-\mathbf{b}\|_{2}
$$


This problem is almost always ill-posed, i.e. the solution is not unique or it is not a continuous function of the data [35]. The problem is also ill-conditioned if the singular values of $\mathbf{K}$ decay gradually to zero and the ratio between the largest and smallest non-zero singular values is large. An arbitrary small perturbation of the data can cause an arbitrarily large perturbation of the solution.

To obtain a stable and physically meaningful result from an ill-posed problem, different variants of the Phillips-Tikhonov (PT) regularization may be considered [32, 34-36]. The PT regularisation with two terms solves the least squares problem:

$$
\mathbf{s}_{\text {reg }}=\arg \min \left(\|\mathbf{K} \mathbf{s}-\mathbf{b}\|_{2}^{2}+\delta\left\|\mathbf{L}\left(\mathbf{s}-\mathbf{s}^{*}\right)\right\|_{2}^{2}+\gamma\left\|\mathbf{H}\left(\mathbf{s}-\mathbf{s}^{*}\right)\right\|_{2}^{2}\right)
$$

where $\delta$ and $\gamma$ and $\mathbf{L}$ and $\mathbf{H}$ are the regularisation parameters and matrices, and $\mathbf{s}^{*}$ is an initial estimate of the solution. The prior information introduced by this regularisation method is smoothness of the solution. The regularisation matrices are responsible for smoothing out the result and the regularisation parameters control the weight of the smoothing. When $\mathbf{b}$ consists of observational data (as opposed to synthetic, forward-modelled data) the measurement covariance matrix $\mathbf{S}$ can be implemented in the solution of Eq. (10) [37, 38]. Moreover, the underlying size distribution cannot take negative values, and therefore a further constraint that can be imposed to Eq. (10) is that all the components $b_{\mathrm{i}}$ of $\mathbf{b}$ are positive. Thus, Eq. (10) can be solved by using a constrained non-negative least squares algorithm.

\subsection{The light scattering model}

The construction of the matrix $\mathbf{K}$ implies assumptions about the properties of the particles used as reference such as size, shape and refractive index. Spheres are the favoured reference geometry due to the straightforward definition of size and the computational feasibility of the scattering models associated to this shape, which are applicable to a specific size and refractive index range (Fraunhofer) or to the full size and refractive index range (Mie). Regarding size, three scattering regimes can be distinguished : Rayleigh regime for $x<2 \pi$, resonance regime for $x \approx 2 \pi$ and geometrical optics for $x$ $>2 \pi$. Note that $r=0.633 \mu \mathrm{m}$ for $x=2 \pi$ and $\lambda=633 \mathrm{~nm}$.

The Fraunhofer model was the first one used by particle sizers [19]. It assumes that the sample is a large (geometrical optics), opaque, non-absorbing disk, i.e. the scattering cross section is equal to the geometric cross section (in other words: all particles scatter with the same efficiency, $Q_{\text {sca }}(x)=1$ ). The forward scattered light originates only from diffraction, and in principle only small scattering angles can be considered. The recommended lower limit for the Fraunhofer approximation is $50 \mu \mathrm{m}(\lambda=633 \mathrm{~nm})$ [19], although empirical studies often find that Fraunhofer can be safely applied for $x>30(r>3 \mu \mathrm{m}$ at $\lambda=633 \mathrm{~nm}$ ) [39]. Mie theory is exact for spheres for the three scattering regimes, since it explicitly calculates the contributions of diffraction, scattering and absorption to the scattering matrix. For this, the complex refractive indices of the particulate samples $\left(m_{\mathrm{p}}=n_{\mathrm{p}}-i k_{\mathrm{p}}\right)$ and the medium where they are suspended $\left(m_{\mathrm{m}}=n_{\mathrm{m}}\right)$ are needed. The scattering efficiency is a function of the size parameter, and the 
real and imaginary parts of the particle-medium relative refractive index $\left(m=\left(n_{\mathrm{p}} / n_{\mathrm{m}}\right)-i\left(k_{\mathrm{p}} / n_{\mathrm{m}}\right)=n-i k\right)$. In the limit of large particle size, the Mie and Fraunhofer optical models converge [40].

Rayleigh scattering is the simplest scattering behavior. It requires particle sizes much lower than the wavelength of the incident and transmitted radiation [25]. As the particle size approaches the Rayleigh regime, the scattering efficiency decreases as the fourth power of $x$ and the phase function tends towards $\cos 2 \theta$ as $x \rightarrow 0$ and thus becomes small, smooth and changes slowly with decreasing size. This means that the columns of $\mathbf{K}$ for $x$ below $\sim 1$ have a very small scaling and are very similar (i.e. correlated, linearly dependent). Such matrix is ill-conditioned and an inversion at these sizes is not feasible. The regularized solution is dominated by the smoothness constraint, producing meaningless results in this size range [16, 34]. This places a lower limit to the accessible range of particle equivalent radii. Performing measurements in the blue part of the spectrum lowers the size limit with respect to usual red wavelength measurements, e.g. for $\lambda=466 \mathrm{~nm}$, the Rayleigh regime is placed below $r=0.06 \mu \mathrm{m}$.

In principle, a numerical model of the interaction between radiation and particles [7,31] combined with a geometrical model of the particles themselves could also be used to generate the columns of the matrix $\mathbf{K}$ for retrieval purposes. Unfortunately, numerical methods are currently limited to a rather reduced range of size parameters for practical reasons. Calculations for $x>50$ are computationally demanding and impractical for retrieval purposes, compared with Fraunhofer and Mie calculations. In addition, the choice of the particle model may differ for different samples (e.g. porous aggregates, compact particles, fractal-like particles, etc), which adds further complexity to the implementation of these methods in particle sizing. It must be highlighted, however, that Fraunhofer and Mie deliver immediate results for a spherical geometry that is rarely displayed by real dust samples.

From Eqs. (6) and (7), it can be anticipated that the discrepancies between the scattering model employed by the LLS method, which uses $\sigma_{\text {sca }}\left(x_{i}\right)$ and $P_{11}\left(x_{i}, \theta_{j}\right)$ calculated for spheres for certain $m$, and the actual scattering behaviour causing the observed or forward-modelled phase functions will be transferred to the retrieved size distribution $\mathbf{s}$. These discrepancies are likely to be dependent on size, since scattering characteristics converge in the large particle limit. Thus, a broad size distribution straddling several scattering regimes may be valid within a certain size range, while it may deviate from the true distribution elsewhere.

\section{Methods}

\subsection{Fraunhofer and Mie computations}

The Mie and Fraunhofer phase functions $P_{11}\left(x_{i}, \theta_{j}\right)$ are the theoretical backbone of the LLS particle sizing method. They are used to fill the columns of $\mathbf{K}$ when one or the other model are selected. The Fraunhofer normalized phase function is given by:

$P_{11}(x, \theta) \propto x^{2}\left(\frac{2 J_{1}(x \sin \theta)}{x \sin \theta}\right)^{2}\left(1+\cos ^{2} \theta\right)$ 
where $J_{1}$ is the Bessel function of the first kind [19]. In this study, Mie phase functions and average scattering cross sections $\sigma_{\text {scat }}(x)$ [25] are calculated with M. Mishchenko's Lorentz-Mie code [31, 41]. Phase functions at $\lambda=633 \mathrm{~nm}$ and $\lambda=466 \mathrm{~nm}$ for a range of radii and angles have been calculated with the two models. The He-Ne laser $(\lambda=632.8 \mathrm{~nm})$ is the staple light source in LLS particle sizing. Some LLS machines perform additional measurements in the blue part of the spectrum, e.g. the Malvern Mastersizer instrument series takes large scattering angle measurements at $\lambda \sim 470 \mathrm{~nm}$. For Mie calculations, a range of relative complex refractive indices is chosen $(1.12 \leq n \leq 2.47,0 \leq k \leq 1)$, considering that many LLS measurements are performed using water as the carrier medium, i.e. $n_{\mathrm{m}}=1.333$ and $1.5 \leq n_{\mathrm{p}} \leq 3.3$. This range encompasses the values of $n_{\mathrm{p}}$ of most mineral dust samples.

A size grid is defined between $r=5 \times 10^{-3} \mu \mathrm{m}$ and $1.1 \times 10^{3} \mu \mathrm{m}$, where the steps between size bins are chosen to be equidistant in a logarithmic scale (steps of 0.06). This configuration is similar to the one used by default by the Malvern Mastersizer 2000 analysis software and is convenient for the usual lognormal character of size distributions. The phase functions for each size bins are averages of many discrete-size phase functions calculated for smaller subdivisions of these bins. Regarding the scattering angle grid, calculations have been performed between $0^{\circ}$ and $180^{\circ}$ in $0.004^{\circ}$ steps. The resolution of the grid in the forward scattering direction is very important, since it determines the retrieval skill near the upper limit of the inversion size range. In this work, a method of angular binning of the phase functions $P_{11}$ emulating the operation of commercial sizers has been implemented. LLS particle sizers reduce the huge dynamical range of the phase functions between forward and side and back scattering (many orders of magnitude) [19] by careful arrangement of the angular distribution and surface area of detectors, which facilitates the inversion. We define here forward scattering as the range of the scattered intensity measured by the so-called focal plane detector array in LLS commercial apparatuses, and side and back scattering as the range measured by large scattering angle detectors. Detectors with annulus sector geometry placed in the focal plane detector array are usually arranged in such a way that they are equidistant in a logarithmic scale. Thus, the logarithm of the detector areas increases with a constant increment, where larger areas balance the much smaller intensities [34, 38]. We apply this in Eq. (5) by integrating the phase functions in a parameterized angular grid encompassing 3 different segments: 33 logarithmic bins between $0.02^{\circ}$ and $5.8^{\circ}, 9$ bins between $7^{\circ}$ and $30^{\circ}$, and 4 bins between $45^{\circ}$ and $135^{\circ}$, emulating the grouping of detectors in a Malvern Mastersizer 2000. To obtain realistic values of the parameters defining the step and size of the bins for each segment, we have carried out the following procedure. The parameters have been varied using a non-linear least squares method until the forwardmodelled phase function of the Malvern Quality Audit Standard 2001 (glass spheres with known size distribution) matches the shape of the phase function measured with a Malvern Mastersizer 2000 apparatus as a function of detector number. The manufacturers of commercial particle sizers usually do not disclose the angular distribution and properties of their detectors.

The Mie $\mathbf{K}$ matrices are also used in this work to carry out forward modelling of phase functions of 
spheres at $\lambda=466 \mathrm{~nm}$ and $\lambda=633 \mathrm{~nm}$ via Eq. (6). for subsequent size distribution retrieval. Because the LLS sizing method is exact for spheres, the results of this exercise are useful to validate the inversion method employed to solve Eq. (7). In addition, this approach can be used to test the sensitivity of the size distribution retrieval to the real and imaginary parts of the refractive index. When using the Mie model in a commercial particle sizer, refractive indices of the particle samples under study need to be supplied. However, in some cases experimental values are not available, and in some other cases they are available, but they are accompanied by relatively large experimental uncertainties. Also, particles consist frequently of mixtures of different materials. Effective medium theories can be used to estimate the effective refractive indices, but they may not provide good estimates for irregular particles.

A range of power law and modified log-normal number size distributions are considered to check the performance of the inversion method across the size parameter range $0.05<x<5000$, i.e. $0.005 \mu \mathrm{m}<r$ $<500 \mu \mathrm{m}$ for $\lambda=633 \mathrm{~nm}$. The so-called modified log-normal $n(r)$ distribution corresponds to a lognormal $S(\log r)$ distribution (see equation 5.247 in [31]). These are the most common distributions of particulate samples that have been previously size-segregated using a combination of test sieves. [42].

To emulate the operation of a commercial sizer, we fill the elements of $\mathbf{b}$ in Eq. (6) primarily with the 46 angular bins at $\lambda=633 \mathrm{~nm}$ and append to these 4 additional elements at $\lambda=466 \mathrm{~nm}$ in the large scattering angle sector (between $45^{\circ}$ and $135^{\circ}$ ). This is expected to provide extended sensitivity near the Rayleigh limit. The vectors b obtained in this way are then inverted (Eq. 9) to estimate of the distribution used originally to generate $\mathbf{b}$ and analyse the quality of the retrieval. In order to keep the number of unknowns equal or smaller than the number of observations (46 angular detectors), the original size bin distribution ( 89 bins) needs resampling if the complete size range is considered in the retrieval (e.g. for power law and broad log-normal distributions). We have also implemented a method to find the optimal retrieval size range as determined by the lowest norm of the phase function fit residuals, which is useful to retrieve very narrow log-normal size distributions. In general, the results in the full and restricted range match well, and the only difference is the higher sampling in the restricted range.

\subsection{Discrete dipole approximation and particle generation algorithm}

In order to study the performance of the LLS method with irregular particles, we have chosen the agglomerated debris and pocked spheres models [43, 44], whose light scattering response has been calculated with the discrete dipole approximation (DDA) technique. The implementation of the DDA method and the particle generation model employed in this work have been described in detail elsewhere $[43,44]$. Agglomerates debris appear to be a more realistic model of mineral dust samples than spheroids or gaussian random spheres [13, 45]. Moreover, agglomerated debris and pocked spheres are equidimensional. Particles with extreme aspect ratios show enhanced scattering at intermediate angles and reduced backscattering [7]. The ISO recommendation is using the LLS technique with particles with aspect ratios lower than 1:3 [19]. Elongated particles may also produce scattering patterns affected by 
flow particle alignment [46], which is beyond the scope of this study. Here it is assumed that particles are presented to the probe laser in all possible orientations. Thus, our study deals with the averaged effect of irregular shape.

Agglomerated debris have a packing density of $\rho=0.236$ (packing density defined as the ratio of particle volume to volume of the circumscribing sphere). The geometric cross section of these particles is on average a factor of 0.61 of the projected area of the circumscribing sphere [44, 47]. In addition, other model particles with higher average packing density $(\rho=0.336)$ have been considered, namely pocked spheres [47]. For these particles, the average ratio of the geometric cross section to the projected area of the circumscribing sphere is 0.79 . Four examples of agglomerated debris and pocked spheres with different morphologies are shown in Figure 1.

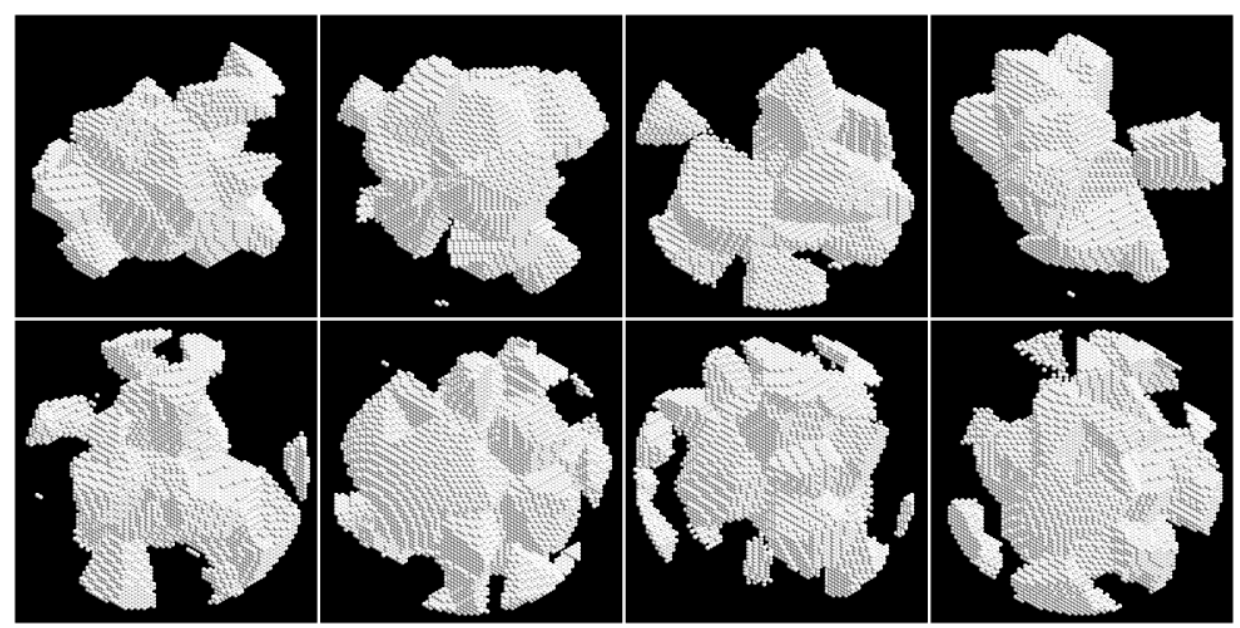

Figure 1. Example images of model agglomerate particles with various morphologies. Top panel row: agglomerated debris. Bottom panel row: pocked spheres

Size is usually defined in relation to the specific method used to generate particles. The size parameter range used in the construction of agglomerated debris and pocked spheres is $1<x^{\prime}<32$, where $x^{\prime}$ corresponds to the radius of the circumscribing sphere of the model particle. Thus, agglomerated debris and pocked spheres with the same $x^{\prime}$ are circumscribed by spheres of the same radii. However, it has been noted before [48] that defining a common measure of size-equivalence for non-spherical particles is not straightforward. Comparing the phase functions of particles with an inconsistent definition of size makes it difficult to distinguish the dependence on size and morphology. LLS sizing provides a common characterization of size defined according to the angular dependence of the phase function of reference particles, which for practical reasons are chosen to be spheres. Thus, for consistency with the radius of spherical particles of the Mie optical model, we will refer hereafter to the size parameter $x$ defined in terms of the radius of the equivalent circular projected area or geometric cross section, which for agglomerated debris is $x=\sqrt{ } 0.61 x^{\prime}=0.78 x^{\prime}$ and for pocked spheres is $x=\sqrt{ } 0.79 x^{\prime}=0.89 x^{\prime}$. Thus, the size parameter ranges defined in this way are respectively $0.78<x<25$ and $0.89<x<28$.

A dataset of light scattering computations with the DDA technique has been used, consisting of phase functions for a range of refractive indices representative of various constituent materials [43, 44, 47]. 
For each set of $x$ and $m$, the light-scattering responses of model particles were averaged over sample particles and their orientations. A minimum of 500 sample particles were considered [47]. The forwardmodelled average phase functions are generated by averaging the phase functions of the model particles in the aforementioned size parameter ranges using selected size distributions via Eq. (6). For averaging, radius is computed from $x$ and $\lambda$ (for agglomerated debris $0.052 \mu \mathrm{m}<r<1.756 \mu \mathrm{m}$ and for pocked spheres $0.055 \mu \mathrm{m}<r<2.005 \mu \mathrm{m}$, at $\lambda=442 \mathrm{~nm}$ ). The phase functions are calculated between $0^{\circ}$ and $180^{\circ}$ in $1^{\circ}$ steps. Since the particles are relatively small, the poorer resolution of the scattering angle grid $\left(1^{\circ}\right)$ is not critical. Degradation of the angular resolution by emulating detector binning is not necessary, since owing to the small size of the particles the differences between the forward peak intensity and side scattering intensities in the fitting range $\left(0^{\circ}<\theta<50^{\circ}\right)$ are within 3 orders of magnitude or lower. Larger scattering angles $\left(\theta>50^{\circ}\right)$ are excluded to avoid sphere-specific features. Most calculations are carried out for $\lambda=442 \mathrm{~nm}$, which is expected to extend the sensitivity to small particles. Some examples are shown in Figure 2, where the effects of size and refractive index are highlighted. It can be seen that the size distribution has an impact over the full scattering angle range for irregular particles with the same optical properties, while the effects of refractive index are confined to $\theta>10^{\circ}$.

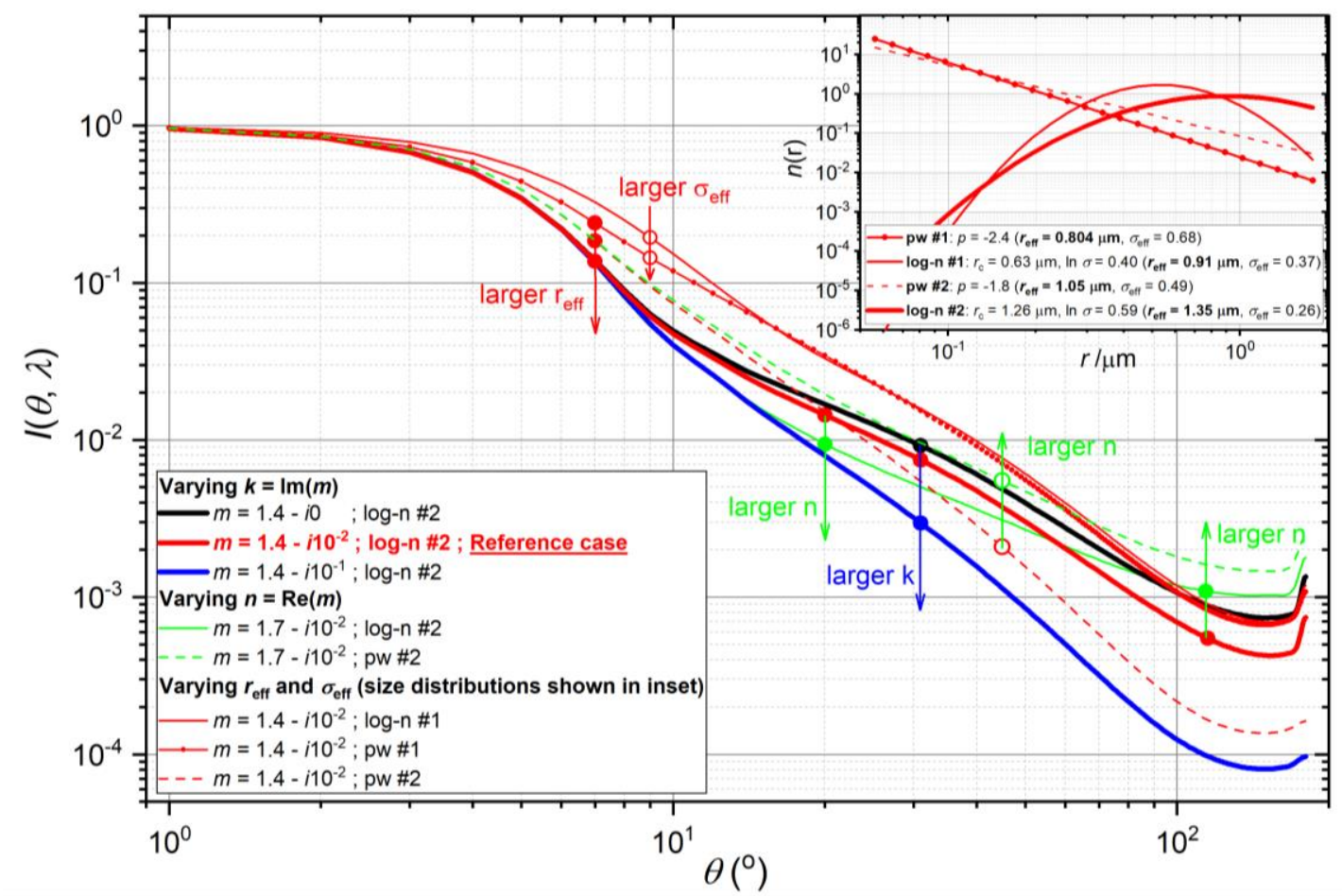

Figure 2. DDA-generated average phase functions (vector $\mathbf{b}$ in Eq. (6)) of eight ensembles of agglomerated debris with different relative refractive indices $m(n=\operatorname{Re}(m), k=\operatorname{Im}(m))$ and size distributions, at $\lambda=442 \mathrm{~nm}$. The log-normal $(\log -n)$ and power law ( $p w$ ) distributions considered are shown in the inset, with their corresponding definition parameters $r_{\mathrm{c}}$ and $\ln \sigma$ and effective parameters $r_{\text {eff }}$ and $\sigma_{\text {eff }}$-see below). The arrows highlight the effect of varying the size distribution and the optical parameters with respect to the reference case (thick red line). The empty circles highlight the different effect of increasing $n$ when the size distribution includes smaller particles.

\subsection{Inversion techniques}

The golden standard of regularized linear inversion techniques is the PT method [36]. The literature on regularization techniques and their applications to particle sizing is extensive. Performing a 
comprehensive test on the different methods available is out of the scope of this work. A major critique to the PT method is that the smoothness assumption cannot be applied in situations where good size resolution is important [49]. More developed non-linear iteration methods exist, which are capable of retrieving non-smooth and quasi-monodisperse distributions [50]. Several commercial instruments are known to implement such numerical methods (e.g. the Sequoia LISST series [51]). However, the distributions measured for laboratory dust samples are usually polydisperse and smooth [42, 52]. In this work we focus on these types of distributions and therefore the validity of the smoothness assumption of the PT method is granted. It must be pointed out, however, that modelling of averaged light scattering quantities of irregular particles using DDA is restricted to a limited size range, which implies artificial sharp cut offs of some of the assumed size distributions.

A regularization toolbox for Matlab [53] has been employed in this work. The PT algorithm with one regularization term has been modified to include a second regularization term and to solve expression (10) using the standard non-negative least squares Matlab routine [54]. The optimal regularisation parameters are estimated using the L-curve corner method and the generalized cross-validation method. The L-curve is a plot for all valid regularization parameters of the semi-norm $\left\|\mathbf{L} \mathbf{s}_{\text {reg }}\right\|_{2}$ of the regularized

solution $\mathbf{S}_{\text {reg }}$ versus the corresponding residual norm $\left\|\mathbf{K} \mathbf{s}_{\text {reg }}-\mathbf{b}\right\|_{2}$. The L-curve displays the compromise between minimization of these two quantities. A log-log plot of the L-curve has a characteristic L-shape, where the point of maximum curvature corresponds to the optimal regularisation parameter. The generalized cross-validation method is based in the idea that if an element $b_{i}$ of $\mathbf{b}$ is ignored, the corresponding regularized solution should predict this observation, and the choice of regularization parameter should be independent of an orthogonal transformation of $\mathbf{b}$. This leads to an optimal regularization parameter that minimizes the so-called generalized cross-validation function. (see [53] and references therein for more details).

\section{Results}

\subsection{Sizing of spheres}

\subsubsection{Validation of the inversion method and intrinsic limitations}

To generate b, power law number size distributions $n(r) \propto r^{p}$ (i.e. $S(\log r) \propto r^{p+3}$, see section 2.2) with 6 different values of $p(p=-1.5,-2,-2.5,-3,-3.5,-4)$ and $\log$-normal size distributions $S(\log r)$ with 10 different values of the location parameter $r_{\mathrm{c}}\left(0.2 \leq r_{\mathrm{c}}<100\right)$ and, for each $r_{\mathrm{c}}, 10$ different values of the scale parameter $\ln \sigma(0.1 \leq \ln \sigma \leq 1)$, have been considered. The retrievals have been performed for size ranges of different width, within the $0.005 \mu \mathrm{m}<r<1100 \mu \mathrm{m}$ range.

Power laws provide mixtures of particles with sizes spanning the full size range considered, and therefore allow to probe the skill of the LLS method in detecting particles with very different sizes. Figure S1, left panel column, shows inversion fits of average phase functions with power laws (red: 633 
$\mathrm{nm}$, blue: $466 \mathrm{~nm})$. The right panel column shows the original $S(\log r)$ (circles) and retrieved (solid thick lines) size distributions. The method retrieves well the size distributions for $r>0.1-0.2 \mu \mathrm{m}$ even for the distributions dominated by large particles. When sharp cut offs are introduced in the power law distributions, the edges of the retrieved distributions are slightly smoothed out as expected (Figure S2).

Modified log-normal size distributions provide scenarios closer to actual measurements performed with particle sizers on size-segregated samples. They allow visualizing how the retrievals deteriorate as the centre of the distribution is shifted towards smaller radii, without the interference of much larger particles that tend to dominate the average phase function. The results become noisier as the tails of the distributions extend below $r \sim 0.1 \mu \mathrm{m}$ and the values of the distribution below this limit are wrong. Despite the deterioration for $r<1 \mu \mathrm{m}$, reasonable values are obtained down to about $0.1 \mu \mathrm{m}$, even if part of the distribution falls below $0.1 \mu \mathrm{m}$. Figure $\mathbf{S 3}$ shows examples of the original (circles) and retrieved (solid thick lines) log-normal size distributions for 3 values of $\ln (\sigma)$. The diagonal values of the averaging kernel $\mathbf{A}=\mathbf{K D}$ (matrix product of the $\mathbf{K}$ matrix by its generalized inverse [34]) are a good indication of the sensitivity of an inversion to a particular range of the independent variable, and in our problem non-zero diagonal values terminate sharply at $r \sim 0.1 \mu \mathrm{m}$.

Of course, if the scattering angle range of $\mathbf{b}$ is reduced by removing large scattering angle bins, the sensitivity to small particles changes. The retrieved $S(\log r)$ is a good estimate of the original one e.g. for $r>4 \mu \mathrm{m}$ when $0^{\circ}<\theta<1^{\circ}, r>0.7 \mu \mathrm{m}$ when $0^{\circ}<\theta<6^{\circ}$, and $r>0.2 \mu \mathrm{m}$ when $0^{\circ}<\theta<30^{\circ}$. These lower size limits are determined by inverting within different angular ranges a forward-modelled phase function obtained by averaging with a power law encompassing the whole size range $(p=-3.5)$. Conversely, if small scattering angle bins are removed, sensitivity to large particles is lost, e.g. $r<10$ $\mu \mathrm{m}$ for $\theta>3^{\circ}$.

The level of noise in the observational data has a critical effect on the results. We have performed size distribution retrievals like those described above for log-normal and power law original distributions, by adding different levels of noise to the simulated detector bins (from $1 \%$ to $10 \%$ of the detector intensity). A $2 \%$ level of noise already has an impact on retrievals for $r<1 \mu \mathrm{m}$. The retrieval in the large particle range is more robust. By adding $8 \%$ all the retrievals shown are affected to some extent either by noise in the retrieved size distribution or by the apparition of spurious modes in the small particle end. The problem of noise is addressed by commercial analysers by considering the measurement covariance matrix $\mathbf{S}$ in the solution (Eq. 13). This is however problematic since the detectors showing more fluctuations are usually those placed at wider scattering angles, i.e. those most sensitive to the small particle range. For example, in the Malvern Mastersizer 2000 instrument the detectors with $\theta>2^{\circ}$ show larger intensity fluctuations and receive accordingly lower weights in the retrieval. Thus, weighting helps in removing noise from retrievals of size distributions ranging above a few microns (e.g. [38]), but may reduce sensitivity to small particles compared to large particles. 

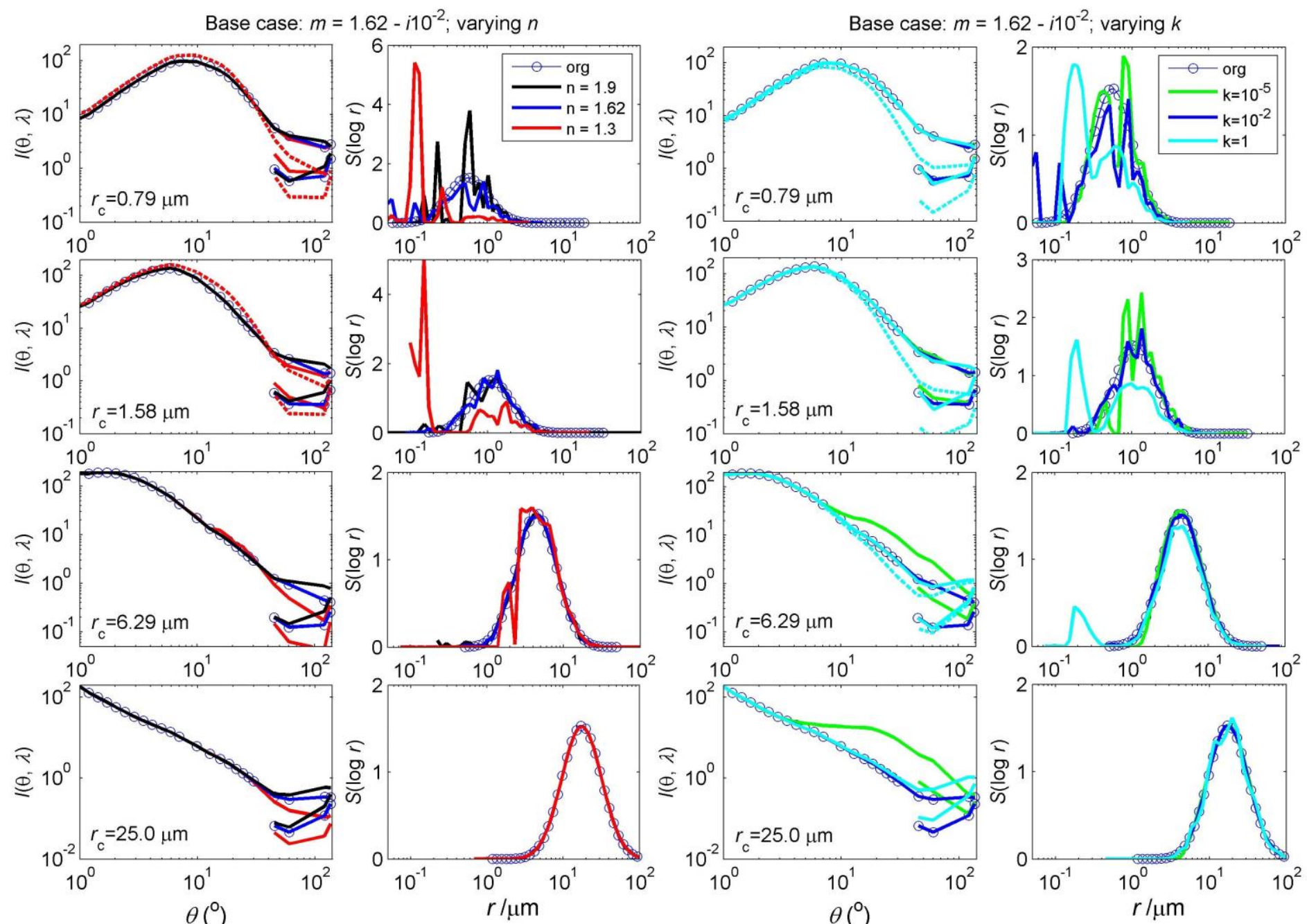

Figure 3. Variation of the angle-binned average phase function and retrieved size distribution by changing the refractive index in the inversion model for spheres. The original distributions are $\log$-normal with $\ln \sigma=0.6$ and $r_{\mathrm{c}}=0.79 \mu \mathrm{m}-25 \mu \mathrm{m}$. Two panel columns on the left: varying the real part of the refractive index $(n)$; two panel columns on the right: varying the imaginary part of the refractive index $(k)$.Circles: forward-modelled average phase functions with $m=1.62-i 10^{-2}$ and original distributions. The fitted average phase functions and retrieved distributions are shown by solid lines (black: $m=1.62-i 10^{-2}$ in retrieval model; red: $m=1.3-i 10^{-2}$; green: $m=1.62-i 10^{-5}$; cyan: $m=1.62-i 1$; blue: true refractive index $m=1.62-i 10^{-2}$ ). In the average phase function panels, the 4 low-lying points correspond to $466 \mathrm{~nm}$, and the rest are for $633 \mathrm{~nm}$. The dashed lines are forward-modelled phase functions using the original size distribution and the values of $n$ and $k$ indicated in the legend. 


\subsubsection{Sensitivity to refractive index}

Deviations of the retrieved size distribution from the true distribution employed in forward modelling can be investigated by constructing the $\mathbf{K}$ matrix with the Fraunhofer approximation or with Mie theory using refractive indices deviating from the true one. We assume that the refractive index is wavelengthindependent in the visible spectral range. To evaluate the effect of using a 'wrong' refractive index, we compare the size distributions retrieved with these to the original ones, besides the phase function fit quality. We also compare the Mie forward-modelled size distributions obtained with the true and the perturbed indices, both for the original size distribution, in order to identify the scattering angle range where most discrepancies occur.

First, the Fraunhofer model has been used to retrieve size distributions from average phase functions with different refractive indices in the scattering angle range $0^{\circ} \leq \theta \leq 30^{\circ}$. We avoid the third segment of emulated detectors at $45^{\circ}, 60^{\circ}, 120^{\circ}$ and $135^{\circ}$, since Fraunhofer's phase functions deviate significantly from Mie for the first two angles of this set and are not defined for the last two. This range gives sensitivity to sizes down to $r \sim 0.2 \mu \mathrm{m}$ with Mie as mentioned above, but it excludes the $466 \mathrm{~nm}$ data. We have found that the lower limit of the size range can be as low as $0.5 \mu \mathrm{m}$ provided that the particles are very absorbing $(k=1)$ and with a high real part of the refractive index $n$. By contrast, the enhanced side scattering characteristic of transparent spheres is balanced by fitting an extra diffraction mode of particles with radii $r \leq 1 \mu \mathrm{m}$ (a spurious mode usually observed in distributions retrieved with commercial particle analysers). Note that the Mie scattering efficiency $Q_{\text {scat }}(r)$ is generally underestimated by the Fraunhofer approximation $\left(Q_{\text {scat }}(r)=1\right)$, except for high absorption, and that the maximum discrepancy, occurring at the first resonance peak, shifts to larger sizes as the real part of the refractive index becomes smaller $[19,25]$. In general, it is safe to use the Fraunhofer approximation for $r>3 \mu \mathrm{m}$ (lowest valid size for a $\lambda=633 \mathrm{~nm}$ forward-modelled phase function with $m=1.12-i 10^{-5}$ ) when the true refractive index of the spheres is unknown. Fraunhofer can be considered as the particular case of assuming high values of the real and imaginary part of the refractive index in the inversion retrieval (flat $Q_{\text {scat }}$ practically for $r>0.1 \mu \mathrm{m}$ ). For consistency with the Mie calculations, we have repeated these Fraunhofer retrievals from forward-modelled phase functions at $\lambda=466 \mathrm{~nm}$ with the same angular bins. As expected, the lower limits are reduced by a factor $466 / 633 \sim 0.74$, and therefore the Fraunhofer retrievals are valid for $r>2.2 \mu \mathrm{m}$ if a particle sizer uses a $\lambda=466 \mathrm{~nm}$ light source.

In order to study the impact of a poor guess of the refractive index used in the optical model on the size distribution retrieval, we have chosen as our reference case $m=1.62-i 10^{-2}$. We use the full scattering angle range $\left(0^{\circ}<\theta<135^{\circ}\right)$ and the usual two wavelengths $466 \mathrm{~nm}$ and $633 \mathrm{~nm}$. Figure 3 (second panel column from the left) shows that underestimating the real part of the refractive index $(n=1.3)$ results in oscillations in the retrieved distribution and an enhancement or 'spurious mode' between $0.1 \mu \mathrm{m}$ and 1 $\mu \mathrm{m}$, when there are particles with sizes below $1 \mu \mathrm{m}$. As shown by the comparison of forward-modelled phase functions, for low $n$ (dashed red line in first panel column from the left) there is less scattered 
intensity for $\theta>40^{\circ}$ than for higher $n$ (circles), which is balanced in the fit (solid red line) by additional small particles. Overestimating the real part $(n>1.7)$ generates noisier retrievals and 'holes' in the retrieved distribution when the original size distribution encompasses a significant fraction of particles with $r<1 \mu \mathrm{m}$. Overestimating of the imaginary part of the refractive index has a similar effect to underestimating the real part, and results in a spurious mode appearing between 0.1 and $1 \mu \mathrm{m}$. (Figure 3, fourth panel column from the left). For wide distributions like the log-normal in Figure 3, this spurious mode may overlap with the genuine mode. Underestimating the extinction coefficient by one order of magnitude or more causes oscillations and holes, similar to overestimating $n$. The excess side scattering produced by low $k$ when particles are large (forward model for $k=10^{-5}$, green lines in third panel column from the left) does not have a significant impact on the retrieved distribution, which is dominated by fitting of the forward peak.
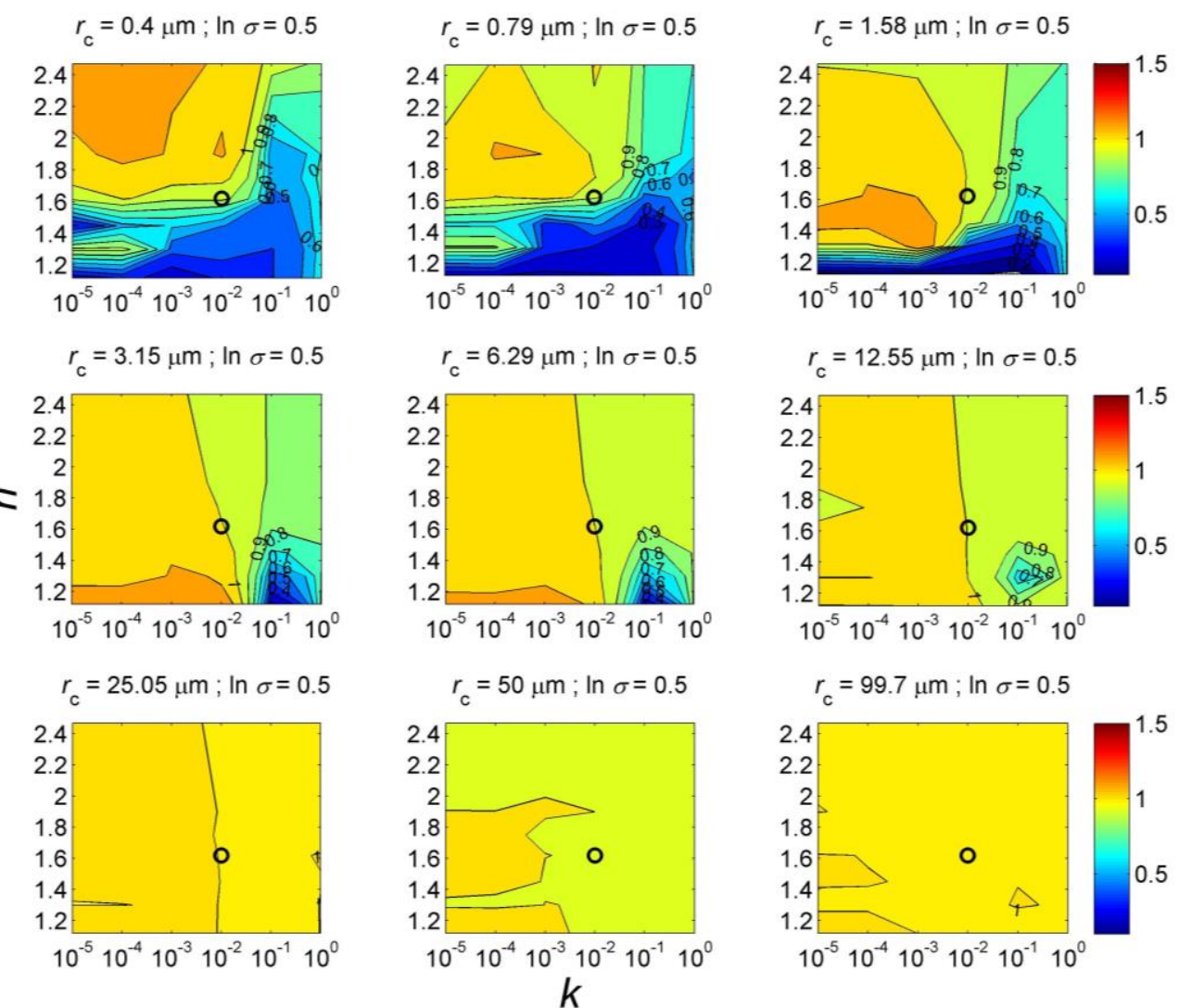

Figure 4. Comparison between the effective radius of original distributions of spheres used in the Mie forward-modelling of the average phase function $\left(m=1.62-i 10^{-2}\right)$ and the effective radius of the distributions retrieved by inversion using Mie with different values of the real and imaginary parts of the refractive index. The original distributions are log-normal with $r_{\mathrm{c}}$ between 0.4 and $99.7 \mu \mathrm{m}$ and $\ln \sigma=0.5$. The contour plots show the ratio of the effective radius of the retrieved distribution to the one of the original distribution $\left(r_{\text {eff }}(\right.$ retrieved $) / r_{\text {eff }}$ (original). The circle corresponds to the retrieval performed with the correct $m$.

Figure 4 shows contour plots of $r_{\text {eff }}$ (retrieved) $/ r_{\text {eff }}$ (original) for log-normal distributions with $r_{\mathrm{c}}$ between 0.4 and $99.7 \mu \mathrm{m}$ and $\ln \sigma=0.5$. The result of the retrieval with the correct complex refractive index is indicated by a circle. These contour plots summarize the main features of the sensitivity of the LLS method to the value of the complex refractive index chosen to perform the inversion: large radii whose tail does not reach below $1 \mu \mathrm{m}$ (Figure 3 and Figure 4, bottom rows). 
(ii) sensitivity limits appear around $n \sim 1.5$ and $k \sim 0.05$ (Figure 4, top panel row), receding as the distribution shifts to larger sizes (Figure 4, middle row). The retrieval is insensitive to $n$ and $k$ for size distributions peaking at large sizes (Figure 4, bottom row)

(iii) underestimation of $n$ and overestimation of $k$ result in enhanced $S(\log r)$ (spurious size modes) for $r<1 \mu \mathrm{m}$ (Figure 3, first, second and third panel rows), i.e. distributions with smaller $r_{\text {eff }}$ (Figure 4, top panel row) and conversely a larger $\sigma_{\text {eff. }}$

(iv) overestimation of $n$ and underestimation of $k$ result in holes in the retrieved distribution for $r<1 \mu \mathrm{m}$, i.e. narrower distributions with larger effective radius (Figure 4, top panel row)

(v) the retrieval shows less sensitivity to overestimation of $n$ and underestimation of $k$, as shown by the upper left corner of the contour plots in Figure 4.

(vi) the equivalent results to applying the Fraunhofer approximation is placed at the top right corner of each panel in Figure 4. Overestimation of $k$ by using Fraunhofer is partially phased out by overestimating $n$, implying that using Fraunhofer may give better results than making a too low guess of $n$.

Similar contour plots to those in Figure 4 with opposite behaviour are obtained for $\sigma_{\text {eff }}($ retrieved $) / \sigma_{\text {eff }}$ (original): an enhancement at the small particle end means a wider distribution with a smaller effective radius and larger effective standard deviation.

Using mid-range values of $n$ and $k$ in the forward model, i.e. $m=1.62-i 10^{-2}$, enables seeing well the effect of underestimating $n$ and overestimating $k$, but not so well the effect of overestimating $n$ and underestimating $k$. In order to visualize the effect of using values of $n$ and $k$ respectively in excess and defect of the real ones, forward modelling has been carried out with a power law with $p=-4$ for the cases $m=1.12-i 10^{-2}$ and $m=1.62-i 10^{-5}$, and the retrievals have been performed using $m=1.12-i 10^{-2}$. We have chosen this particular exponent because such power law is dominated by particles with sizes within the range where the largest discrepancies between $Q_{\text {sca }}$ curves for different refractive indices occur. A log-normal centred within such range and sufficiently wide would be equally valid for this exercise. Figure 5 (top row) shows that using the correct refractive index produces very good estimates of the power law for $r>0.1 \mu \mathrm{m}$. Forward modelling using $n=1.12$ and inversion with $n=1.62$ produces a collapse in the estimated size distribution below $2 \mu \mathrm{m}$ (red line in Figure 5a, while the opposite produces an enhancement (cyan line in Figure 5a). Comparison of the $Q_{\text {sca }}$ curves in Figure $\mathbf{5} \boldsymbol{b}$ and $P_{11}$ contours in Figure $5 \boldsymbol{c}$ readily explains such behaviour by a balance between $Q_{\text {sca }}$ and $P_{11}$ in the inversion model and the $S(\log r)$ estimate. Note that in the size range where the mismatch between the $Q_{\text {sca }}$ curves occurs there is also a mismatch in the same direction of the $P_{11}$ contours. Similarly, forward modelling using $k=10^{-1}$ and inversion with $k=10^{-5}$ results in a collapse of the estimated distribution below $r \sim 0.5$ $\mu \mathrm{m}$ (red line in Figure 5d), while the opposite results in an enhancement (cyan line in Figure 5 $\boldsymbol{d}$ ). In this case the mismatch between $Q_{\text {sca }}$ and the $P_{11}$ contours is less severe, which explains why the retrievals in Figure $\mathbf{5} \boldsymbol{d}$ with wrong $k$ are not as bad as those in Figure $\mathbf{5} \boldsymbol{a}$ with wrong $n$. 

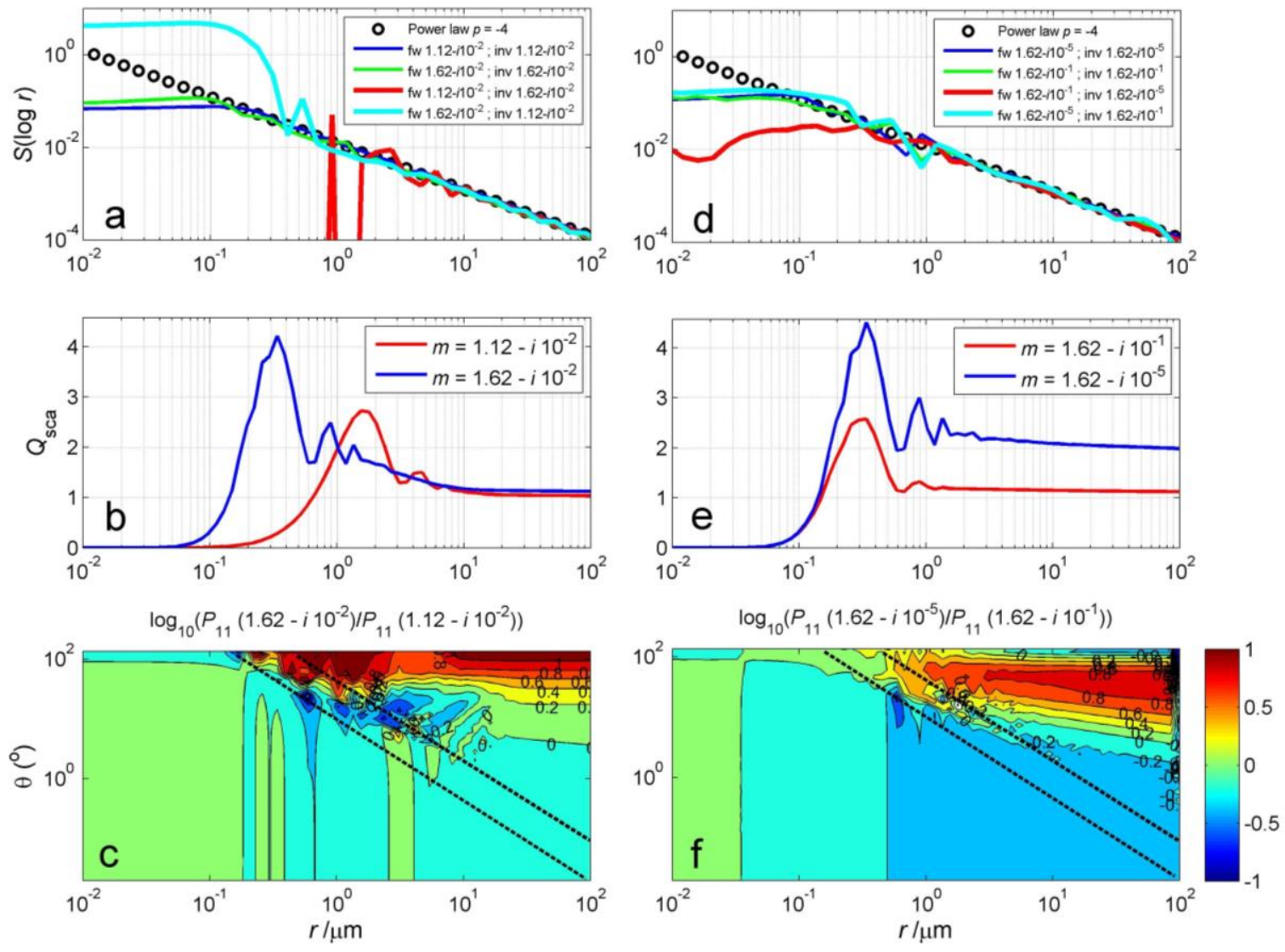

Figure 5. Left panel column: size distribution retrievals using very different values of $n$. Panel $a$ : original power law $(p=-4)$ and retrieved size distributions (blue: $m=1.12-i 10^{-2}$ forward and inversion; green: $m=1.62-i 10^{-2}$ forward and inversion; red: $m=1.12-i 10^{-2}$ forward and $m=1.62-i 10^{-2}$ inversion; cyan: $m=1.62-i 10^{-2}$ forward and $m=1.12-i 10^{-2}$ inversion. Panel $b$ : $Q_{\text {sca }}(r)$ for $m=1.12-i 10^{-2}$ and $m=1.62-i 10^{-2}$. Panel $c: P_{11}(r, \theta)$ for $m=1.12-i 10^{-2}$ and $m=1.62-i 10^{-2}$. Right panel column: the same for very different values of $k\left(m=1.62-i 10^{-5}\right.$ and $\left.m=1.62-i 10^{-1}\right)$. The dashed lines in panels $c$ and $f$ roughly indicate the angular range of $P_{11}$ which dominates the retrieval for each size.

The examples above are useful because they help visualizing important effects of particle irregularity and porosity such as lower effective $n$ and higher effective $k$, as it will be shown in next section. Such large errors in estimating the refractive index of dust samples can be avoided by using other information such as obscuration (transmission) measurements performed by commercial particle sizers. However, it must be noted that LLS measurements are usually carried out in dispersion media other than air with $n_{\mathrm{m}}$ $>1$, for example water $\left(n_{\mathrm{m}}=1.333\right)$. In such cases the sensitivity contours in Figure $\mathbf{4}$ are compressed towards the low end of the range of the real part of $n$. This is illustrated in Figure $\mathbf{S 4}$ for an extended range of $n: 1.05 \leq n \leq 2.47$ (i.e. $1.4 \leq n_{\mathrm{p}} \leq 3.3$ ). For a particle material with a refractive index of $n_{\mathrm{p}}=1.6$ in water, the relative refractive index becomes $n=1.2$. In this range the sensitivity to small errors in the refractive index is larger, especially if the distribution is dominated by particles in the submicron range. For example, for the $p=-4$ power law discussed above, an uncertainty of $10 \%$ in the refractive index of the particles results in $\sim 20 \%$ errors in $r_{\text {eff }}$ of the retrieved distribution in air, and $50 \%$ errors in water.

\subsection{Sizing of irregular particles}

Despite of the limited size range considered in the modelling of agglomerated debris, this is the range where the effects of shape and refractive index are expected to approach the same order than that of size. 
In order to explore these effects, we have forward-modelled phase functions for spherical particles with Mie theory and irregularly shaped debris agglomerates with the DDA technique (some examples are shown in Figure 2), using the power-law and log-normal size distributions listed in Table 1. The forward-modelled phase functions plotted in Figure 2 show the following characteristics:

(i) The forward peak $\left(\theta<7^{\circ}\right)$, is narrower for larger particles (red dots, larger $r_{\text {eff; }}$, red circles larger $\sigma_{\text {eff }}$ and similar $\left.r_{\text {eff }}\right)$

(ii) Side and back scattering, $\left(\theta>7^{\circ}\right)$, are less intense as $k$ increases (black-red-green dots).

(iii) Side and back scattering $\left(\theta>7^{\circ}\right)$ are more intense as $n$ increases if small particles are dominant (red and green empty symbols).

(iv) when large particles dominate, side scattering $\left(7^{\circ}<\theta<60^{\circ}\right)$ is less intense and back scattering $\left(90^{\circ}<\theta<180^{\circ}\right)$ is stronger as $n$ increases (red-green filled circles).

We have performed size distribution retrievals by regularized inversion from the phase functions obtained with the DDA technique for scenarios in Table 1. In this case we do not apply the same detector area and sensitivity scaling simulation that we applied for spheres, since the model particles are small and as a consequence their phase functions differ by less than 3 orders of magnitude between forward and side scattering. Retrievals are performed with the Mie and Fraunhofer optical models for a single wavelength (442 nm or $633 \mathrm{~nm}$ ) for scattering angles between $0^{\circ}$ and $50^{\circ}$. We have determined in section 4.1.1 that this range provides sensitivity in to sizes down to the $r \sim 0.1 \mu \mathrm{m}$. Retrievals in a wider angular range up to $135^{\circ}$ have also been carried out, but this results in poorer quality fits due to the deep minimum of the phase function for spheres around $\theta=100^{\circ}$ [7]. The parameters of the distributions retrieved with Mie at $442 \mathrm{~nm}$ for $0^{\circ} \leq \theta \leq 50^{\circ}$ are summarized in Table S1. The ratios between the retrieved and original values of $r_{\text {eff }}$ and $\sigma_{\text {eff }}$ are plotted in Figure 6. It is worth emphasizing that the size range considered is narrow and only includes particles with $0.78<x<39$, i.e. in the Mie regime. In our context, 'large' particles are considered to be those with $10<x<39$, and 'small' particles are those with $0.78<x<10$. Table $\mathbf{S 1}$ also lists the parameters of power laws $(p)$ and log-normal distributions $\left(r_{\mathrm{c}}\right.$ and $\ln \sigma$ ) functions fitted to the retrieved distributions within the size interval indicated by $r_{\min }$ and $r_{\text {max }}$. The fitting interval for the analytical distributions has been chosen by removing points at the high and low ends that deviate from the general trend at the centre of the range. It can be seen that both the fitted and the effective parameters are in general in good agreement with those of the original distributions. The trends observed in the effective parameters are discussed below.

This exercise is similar to comparing forward-modelled phase functions and integrated quantities for spheres and irregular particles in order to observe the deviations of the scattering pattern of the later with respect to the former. Thus, we also plot forward-modelled phase functions for spheres obtained using Mie alongside the phase functions of irregular particles and the corresponding Mie inversion fits (see e.g. first panel row of Figure 7). This helps in understanding what type of compensations are forced into the retrieved size distributions when the inversion fits are performed. 
Table 1 Material refractive index and size distributions used in forward modelling of the phase function of clouds of irregular particles from DDA-generated monodisperse phase functions.

\begin{tabular}{lllllllll}
\hline Scenario & $\boldsymbol{n}$ & $\boldsymbol{k}$ & Size distribution $^{\boldsymbol{a}}$ & $\boldsymbol{p}$ & $\boldsymbol{r}_{\mathbf{c}} / \boldsymbol{\mu \mathbf { m } ^ { b }}$ & $\boldsymbol{\operatorname { l n }} \boldsymbol{\sigma}$ & $\boldsymbol{r}_{\text {eff }} / \boldsymbol{\mu m}$ & $\boldsymbol{\sigma}_{\text {eff }}$ \\
\hline 1 & 1.7 & 0 & Power law & -2.7 & & & 0.660 & 0.802 \\
2 & 1.6 & 0.03 & Power law & -3.1 & & & 0.470 & 0.996 \\
3 & 1.6 & 0.15 & Power law (pw \#2) & -1.8 & & & 1.050 & 0.492 \\
$4^{c, d}$ & 1.313 & 0 & Power law & -2.3 & & & 1.260 & 0.661 \\
5 & 1.4 & 0.01 & Power law (pw \#1) & -2.4 & & & 0.804 & 0.677 \\
$6^{e}$ & 1.4 & 0.01 & Log-normal (log-n \#1) & & 0.63 & 0.405 & 0.913 & 0.368 \\
$7^{e}$ & 1.4 & 0.01 & Log-normal (log-n \#2) & & 1.26 & 0.588 & 1.354 & 0.261 \\
$8^{d, e}$ & 1.6 & $5 \times 10^{-4}$ & Log-normal (log-n \#1) & & 0.63 & 0.405 & 0.913 & 0.368 \\
$9^{d, e}$ & 1.6 & $5 \times 10^{-4}$ & Log-normal (log-n \#2) & & 1.26 & 0.588 & 1.354 & 0.261 \\
10 & 1.4 & 0 & Power law (pw \#2) & -1.8 & & & 1.050 & 0.492 \\
11 & 1.4 & 0.01 & Power law (pw \#2) & -1.8 & & & 1.050 & 0.492 \\
12 & 1.4 & 0.1 & Power law (pw \#2) & -1.8 & & & 1.050 & 0.492 \\
13 & 1.7 & 0 & Power law (pw \#2) & -1.8 & & & 1.050 & 0.492 \\
14 & 1.7 & 0.01 & Power law (pw \#2) & -1.8 & & & 1.050 & 0.492 \\
15 & 1.7 & 0.1 & Power law (pw \#2) & -1.8 & & & 1.050 & 0.492 \\
16 & 1.4 & 0 & Log-normal (log-n \#2) & & 1.26 & 0.588 & 1.354 & 0.261 \\
$18^{f}$ & 1.4 & 0.1 & Log-normal (log-n \#2) & & 1.26 & 0.588 & 1.354 & 0.261 \\
19 & 1.7 & 0 & Log-normal (log-n \#2) & & 1.26 & 0.588 & 1.354 & 0.261 \\
20 & 1.7 & 0.01 & Log-normal (log-n \#2) & & 1.26 & 0.588 & 1.354 & 0.261 \\
21 & 1.7 & 0.1 & Log-normal (log-n \#2) & & 1.26 & 0.588 & 1.354 & 0.261 \\
\hline
\end{tabular}

${ }^{a}$ Power law number size parameter distribution: $n(r) \propto r^{p}$; In brackets: abbreviation used in Figure 2.

Log-normal number size parameter distribution $n(r)=\frac{1}{\sqrt{2 \pi} \ln \sigma r} \exp \left(-\frac{\left(\ln r-\ln r_{\mathrm{c}}\right)^{2}}{2(\ln \sigma)^{2}}\right)$

${ }^{b}$ Radius of the geometric cross section.

${ }^{c}$ This particular scenario is defined in a wider size parameter range for agglomerated debris: $0.78<x<39(0.055 \mu \mathrm{m}<r<$

$2.743 \mu \mathrm{m}$ for agglomerated debris at $\lambda=442 \mathrm{~nm}$ )

${ }^{d .}$ Scenarios calculated also for pocked spheres

${ }^{e}$ Phase functions computed for $\lambda=442 \mathrm{~nm}(0.052 \mu \mathrm{m}<r<1.756 \mu \mathrm{m}$ for agglomerated debris $)$ and $\lambda=633 \mathrm{~nm}(0.078 \mu \mathrm{m}<r$ $<2.515 \mu \mathrm{m}$ for agglomerated debris)

${ }^{f}$ Scenario \#17 is the same as scenario \#7

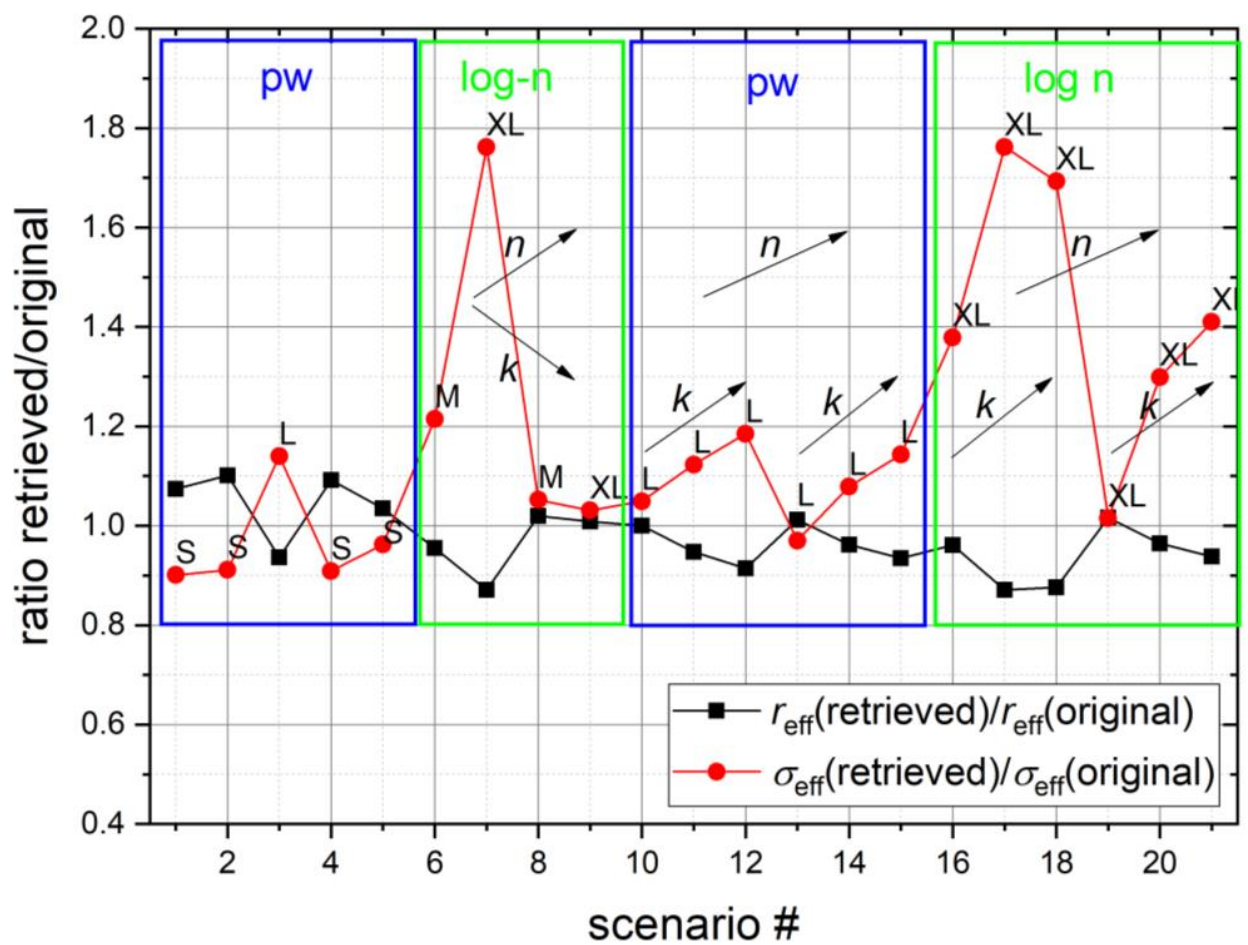

Figure 6. Ratio of effective parameters of retrieved distributions to those of the original ones for each scenario at $442 \mathrm{~nm}$ (black squares: ratio off effective radii; red circles: ratio of effective standard deviations). Distributions dominated by 'small' (steep power laws), 'medium-sized' (peak between $0.3 \mu \mathrm{m}<\mathrm{r}<1 \mu \mathrm{m}$ ), 'large' (shallow power laws) and 'very large' particles (peak at $\mathrm{r}>1 \mu \mathrm{m}$ ) are denoted respectively by S, M, L and XL. The arrows indicate the change directions (increasing or decreasing) of the real and the imaginary part of the refractive index within each block (see Table S1) 

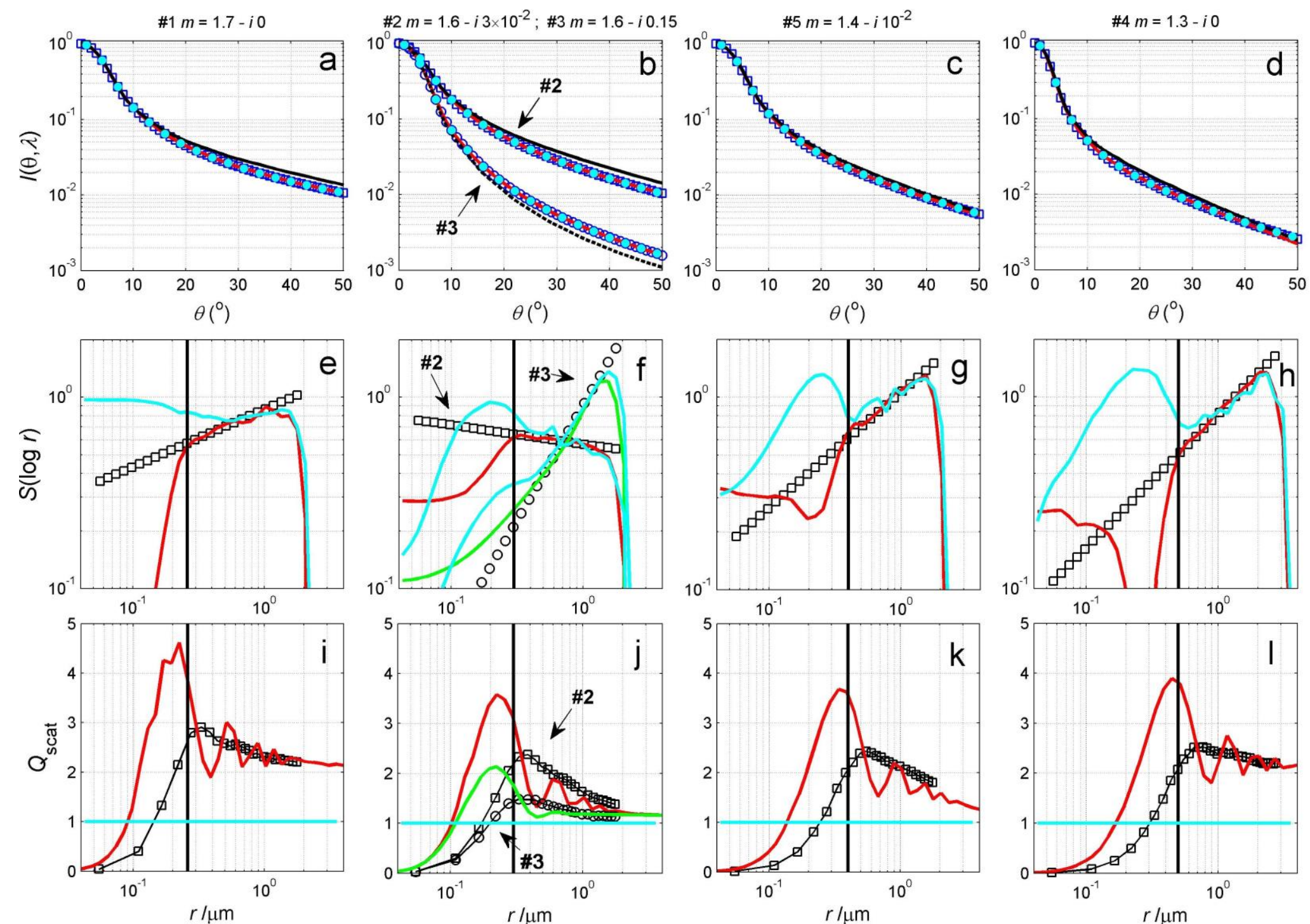

Figure 7. Retrievals of scenarios \#1-\#5 (power laws) sorted by the value of $n=\operatorname{Re}(m)$ in desdending order from left to right. Top panel row: average phase function of agglomerated debris at 442 $\mathrm{nm}$ (blue symbols), Mie inversion fit (red), Fraunhofer inversion fit (cyan symmbols) and average phase function of spheres (black). Middle row panel: original $S$ (log $r$ ) (black symbols) and retrieved $S(\log r)$ (red and green lines Mie, cyan Fraunhofer). Bottom panel row: scattering efficiency of irregular particles (black symbols) and of spheres (red and green lines, cyan Fraunhofer). 


\subsubsection{Entanglement of the dependence of $I(\theta, \lambda)$ on size, refractive index and irregularity}

Figure 7 shows retrievals for scenarios \#1 - \#5 of agglomerated debris. The top panel row shows the forward modelled average phase functions $I(\theta, \lambda)$ for agglomerated debris and for spheres (blue squares and black lines, respectively) and the inversion fits of the former (red lines). According to Eq. (7)-(9), the LLS method uses a Mie (or Fraunhofer) matrix $\mathbf{K}$ for equivalent spheres to fit the average phase function of irregular particles by adjusting the size distribution $S(\log r)$. Since the $I(\theta, \lambda)$ of agglomerated debris and spheres are very close for $\theta<10^{\circ}$, the $S(\log r)$ retrieved from inversion of $I(\theta, \lambda)$ is expected to be close to the original one at the large particle end of the range. This is the case, as shown in the middle panel row of Figure 7, except for the two largest size bins. Regularization smooths the edge of the retrieved distribution, at the size where the distributions employed in forward modelling are truncated due to the computational limitations of DDA calculations. By contrast, the large size wing of the log-normal distribution in scenario \#6, which is not truncated by the upper limit of the size range, is well retrieved (Figure S5). Such sharp cut-offs are not expected in real samples. The smoothing of the cut-off is well understood and therefore we do not attempt to perform a retrieval with improved resolution. Note that the wider definition range of scenario \#4 (higher upper limit $r<2.743 \mu \mathrm{m}$ ) is reproduced, and that the smoothing appears at the higher cut-off of this scenario (compare panels $\boldsymbol{e}-\boldsymbol{f}$ to panel $\boldsymbol{h}$ ). With the Fraunhofer model, results of similar quality to Mie are obtained in the upper size of the range. The Fraunhofer analysis for spheres was done at the typical LLS wavelength of $633 \mathrm{~nm}$, while the results for agglomerated debris in Figure 7 are for $\lambda=442 \mathrm{~nm}$. The lower limit for spheres if the wavelength was $\lambda=442 \mathrm{~nm}$ can be estimated to be at $\sim 3 \mu \mathrm{m} \times(442 / 633)=2.1 \mu \mathrm{m}$. For agglomerated debris good results are obtained down to $\sim 0.8 \mu \mathrm{m}$. Thus, it appears that for irregular particles the lower limit of applicability Fraunhofer approximation is lower than for spheres (see discussion below).

Figure 7 shows that at the small particle end of the size range, the retrieved distributions deviate from the original ones to different degrees (slumps and enhancements can be observed in the $S(\log r)$ distributions). In the inversion, the size distribution that optimises the retrieval needs to counterbalance the mismatch between the forward models for irregular and spherical particles at side scattering angles. As shown by Eq. (6)-(7), the differences between the scattering properties of irregular and spherical particles are condensed in $Q_{\text {scat }}(r, \lambda)$ and $P_{11}(\theta, x)$. These two quantities are fixed as Mie (or Fraunhofer) scattering properties, which are significantly different from those of irregular particles in the small size range, as illustrated by the comparison of $Q_{\text {scat }}$ in the bottom panel row of Figure 7. Such differences between spherical and irregular particles are forced into the retrieved $S(\log r)$.

The four scenarios whose size distributions include significant contributions from small particles are \#1, \#2, \#4 and \#5. Because of the slumps, the effective radius and effective standard deviations of the retrieved distributions for these scenarios are respectively higher and lower than those of the original distributions (Figure 6). The other scenarios have smaller numbers of smaller particles, the slumps are therefore small and their impact on the retrieved distributions are limited. The retrieved distributions for 
scenarios dominated by particles closer to the upper limit of the range show spurious modes at small sizes (see for example scenarios \#6 and \#7 in Figure S5), which generally results in smaller effective radii and larger effective standard deviations.

Figure 6 shows that deviations from the effective parameters of the original distributions are more severe for scenarios with low $n$ or high $k$. Figure 7 shows that as the real part of the refractive index decreases (scenarios $\# 1 \rightarrow \# 2 \rightarrow \# 5 \rightarrow \# 4$ ), the scattering efficiency peak of irregular particles and spheres shifts towards larger sizes. The same happens therefore with the region of mismatch between $Q_{\text {sca }}$ for irregular particles and spheres. This reflects in the slumps appearing in the retrieved size distributions between $0.3 \mu \mathrm{m}$ and $0.7 \mu \mathrm{m}$, which also move towards larger sizes as $n$ decreases (the situation is similar to underestimating the real part of the refractive index for spheres, as shown in Figure 5). The implication is that the lower limit of the retrieval is placed at larger sizes for particles with low $n$. This effect is phased out for larger values of $k$ (Figure 7, scenario \#3). This dependence of the small size end of the retrieved size distribution on refractive index is characteristic of size distributions where small particles dominate, such as steep power laws $(p<-2)$ or log-normal distributions centred at small sizes. By contrast, for scenarios where large particles dominate, the retrieved distributions tend to show enhancements at small sizes (compare \#6 and \#7 in Figure S5). Such enhancements are stronger for scenarios with higher $k$ (compare \#7 and \#9 in Figure S5). Figure 8 summarizes this dependence of the fit results on the true refractive index and the abundance of small particles $(r<0.7 \mu \mathrm{m})$. For a lognormal distribution without large contribution of small sizes, spheres (solid and dashed black lines in Figure 8g-i) scatter on average approximately the same intensity (for $k=0$, panel $\boldsymbol{g}$ ) or lower (for $k>$ 0 , panels $\boldsymbol{h}$ and $\boldsymbol{i}$ )) than agglomerated debris (red and blue symbols) at side angles. This results in an enhancement of the retrieved $S(\log r)$ for small $r$ for scenarios with higher absorption (Figure 8j-l). For a power law distribution with larger contribution of small sizes (Figure 8a-c), spheres scatter higher (for $k=0$ ), approximately the same (for $k=0.01$ ) or lower intensity (for $k=0.1$ ) than agglomerated debris at side angles. This results in the slumps in the retrieved $S(\log r)$ at low $k$ and their absence when the absorption is higher. Also, in Figure 8, the lower limits of the retrievals are generally lower for higher $n$. Figure S5 shows that the lower limits are wavelength dependent: they are lower for $\lambda=442$ $\mathrm{nm}$ than for $\lambda=633 \mathrm{~nm}$.

\subsubsection{Accounting for irregularity with an effective refractive index}

We have carried out a study of the sensitivity of the retrievals to the input values of $n$ and $k$ for all the scenarios at $\lambda=442 \mathrm{~nm}$ in Table 1. For this, we have used in the inversion of each $I(\theta, \lambda)$ all possible combinations of 10 values of $n(1.12<n<2.47)$ and $10 \log$-scale values of $k(0<k<1)$. Such calculations allow to find the complex refractive index that best reproduces the original distribution, and whether this coincides or not with the complex refractive index of the constituent material used in forward-modelling $I(\theta, \lambda)$. Figure 9 shows contour plots of the dependence of several indicators of retrieval success on $n$ and $k$ for scenarios \#4 and \#18. 

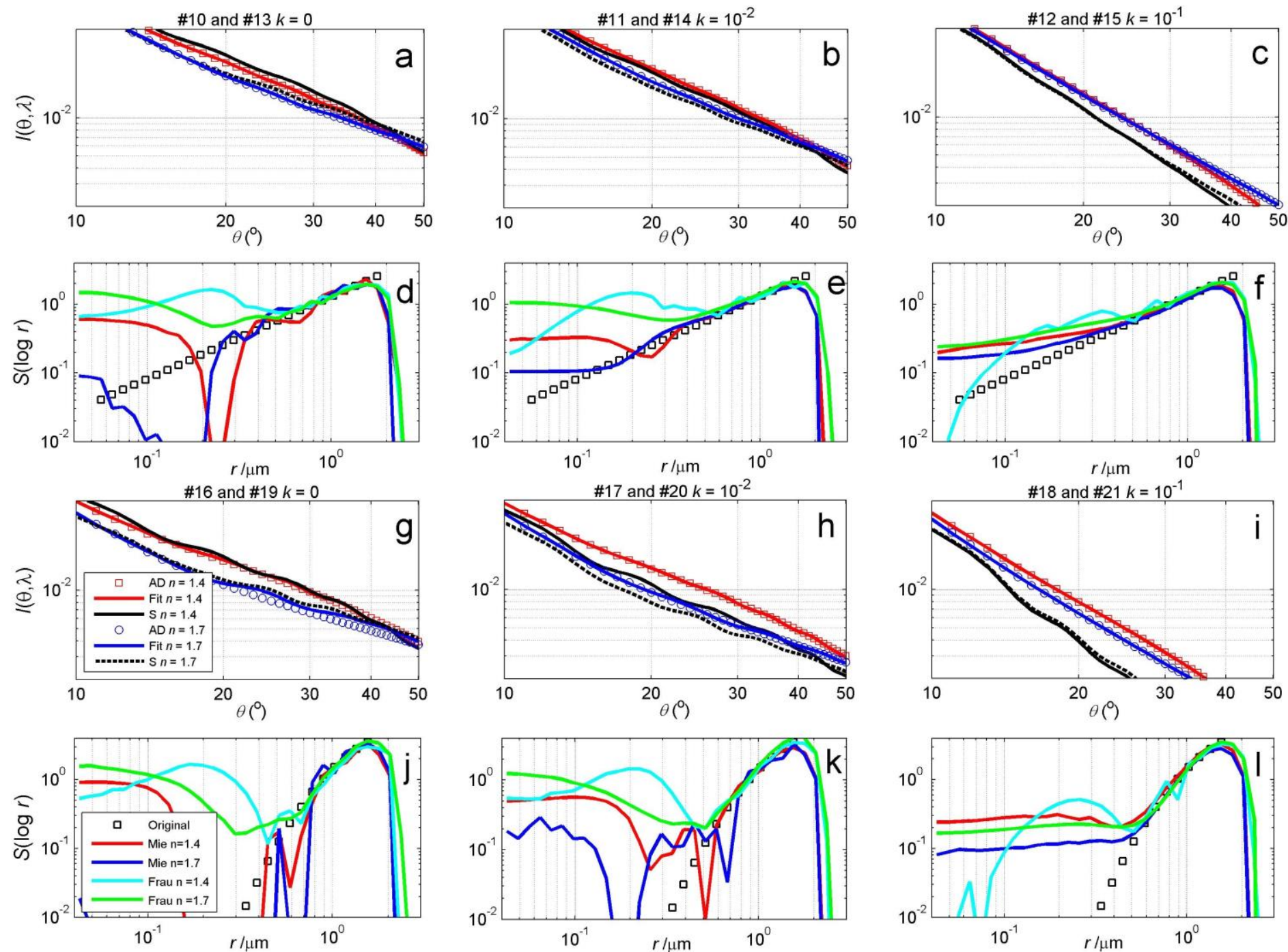

Figure 8 . Retrievals of scenarios \#10-\#15 (power law $\mathrm{p}=-1.8$, panels a $-\mathrm{f}$ ) and \#16-\#21 (log-normal $\mathrm{r}_{\mathrm{c}}=1.26$, panels $\left.\mathrm{g}-1\right)$. Each column corresponds to a value of $\mathrm{k}\left(0,10^{-2}, 10^{-1}\right)$, and each panel contains data for two values of $\mathrm{n}(1.4,1.7)$. Average phase functions between $10^{\circ}$ and $50^{\circ}$ are plotted in the first and third planel rows, including: forward agglomerated debris (AD) for two values of the real part of the refractive index (red squares $n=1.4$ and blue circles $n=1.7)$, inversion fits (red and blue solid lines, respectively) and forward Mie spheres ( $\mathrm{S}$ ) (solid and dashed black lines, respectively). Note that the vertical range of the average phase functions is the same in all panels to facilitate comparison. The original size distributions and the retrieved ones with Mie and Fraunhofer are plotted in the second and fourth panel row. Scenario \#17 is the same as scenario \#7. 
Scenario \#4: $\boldsymbol{m}=1.313-i 0$

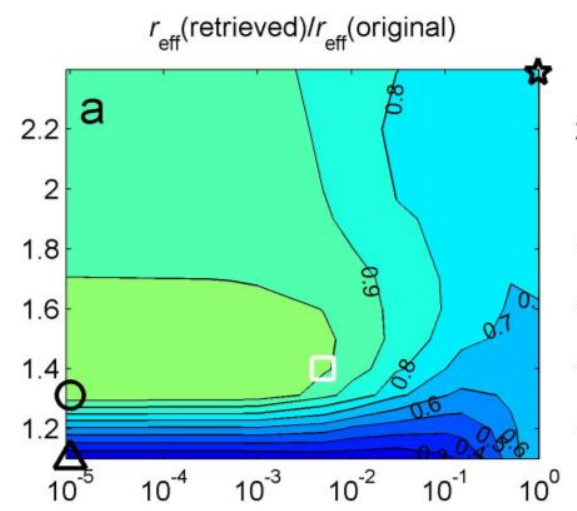

$\log$ MSE distribution (retrieved - original)

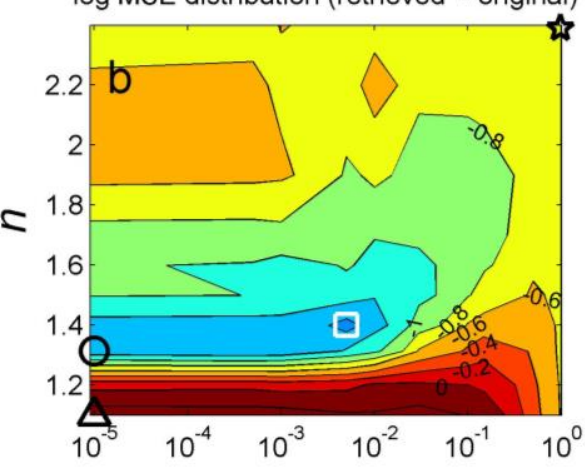

log norm residuals phase function fit

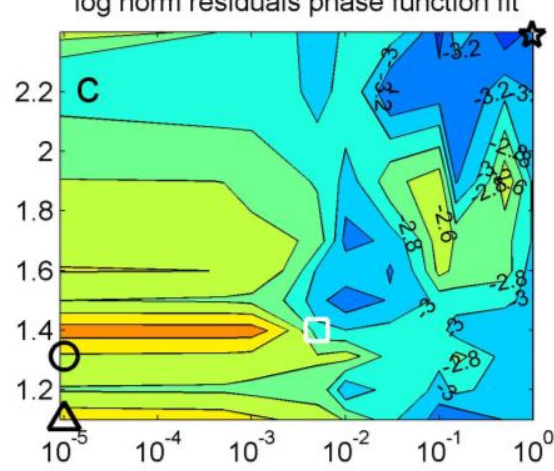

Scenario \#18: $\boldsymbol{m}=1.4-i 0.1$

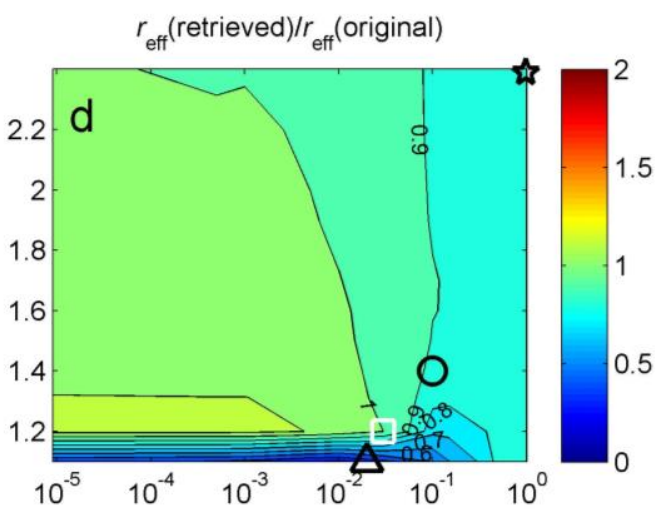

log MSE distribution (retrieved - original)

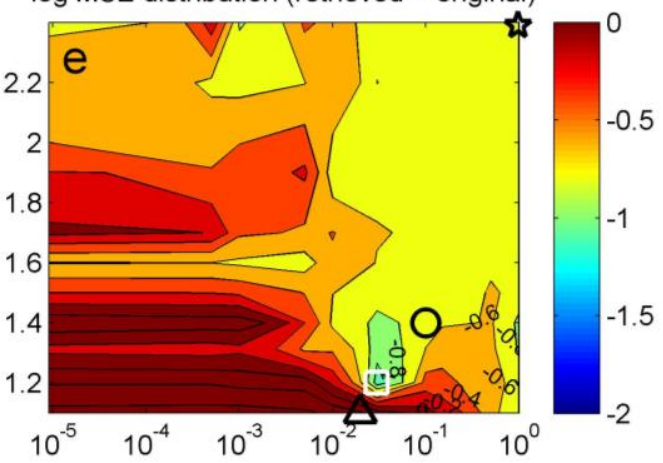

log norm residuals phase function fit

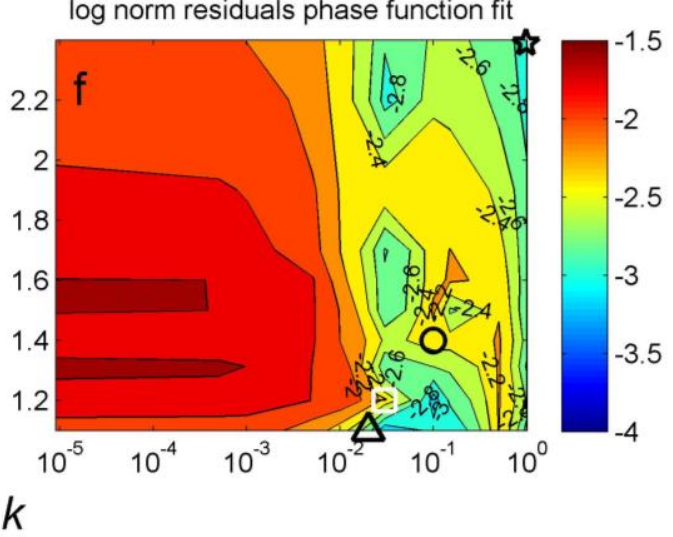

Figure 9. Panels $a$ and $d$ : comparison between the effective radius of the original distribution of scenarios \#4 and \#18 and the effective radius of the distributions retrieved by inversion using Mie with different values of the real and imaginary parts of the refractive index. The contour plots show the ratios of the effective radii of the retrieved distributions to the one of the original distribution. Panels $b$ and $e$ : logarithm of the mean square error of the retrieved distribution with respect to the original one (for $r>0.1 \mu \mathrm{m}$ ) as a function of the values of $n$ and $k$ used in the retrieval. Panels $c$ and $f: \operatorname{logarithm}$ of the norm of the residuals of the phase function fits as a function of the values of $n$ and $k$ used in the retrieval. In the six panels, the circle corresponds to the retrieval performed with the correct refractive index and the white square to the best size distribution retrieval (i.e. the minimum of the surface in panel $c$ ). The result equivalent to Fraunhofer is situated at the upper right corner of each panel (star). The results corresponding to effective refractive indices are marked by triangles.

The contour plot of the ratio of the effective radius of the retrieved distributions to that of the original distribution for scenario \#4 (Figure 9a), is similar to the contour plots in the first panel row of Figure 4 for spherical particles. The retrievals are sensitive to underestimating the real part of the refractive index and to overestimating the imaginary part beyond $k \sim 0.05$. On the other hand, the sensitivity to overestimating $n$ and underestimating $k$ below $k \sim 0.05$ is weaker. In the case of spherical particles, the best result was obtained for the original $m$, while for irregular particles different situations occur 
depending on the true $m$ and size distribution. The best retrieval in terms of similitude to the original distribution for scenario \#4 (white squares) is obtained for a slight overestimation of the real and imaginary parts of the complex refractive index (Figure 9b), which is in the opposite direction than suggested by effective medium approximations (triangles). Effective refractive indices considering the volume fraction of the material with respect to the volume of the circumscribing sphere are listed in Table S2. This pattern is similar for scenarios \#1, \#2 and \#5, with low absorption and important contribution of small particles. By contrast, the region of best results is confined to $n>1.3$ and $k>0.05$ for scenarios with high absorption and dominated by large particles like \#18 (Figure 9d and $\boldsymbol{f}$ ) and \#21. In these cases, better retrievals are obtained by adopting lower values of $n$ and $k$, in the direction indicated by effective medium approximations, but with higher effective $n$.

The Fraunhofer retrieval (star symbols in Figure 9), which is equivalent to using Mie with high $n$ and $k$ in the inversion, also produces reasonable results for $r>0.8 \mu \mathrm{m}$ even for a low $n$ and low $k$ case such as scenario \#4, which is better than when applied to spherical particles. This results from the smooth, almost flat dependence of the scattering efficiency of agglomerated debris to the right of the peak (Figure 7i-l). As for spheres, using Fraunhofer to retrieve the size distribution of irregular particles may yield better results than using Mie with an underestimated value of $n$ (compare contour values for the star and triangle symbols in Figure 9). Interestingly, when the refractive index of agglomerate irregular particles is unknown, it is a safer choice to overestimate $n$ with Mie or by using Fraunhofer than using Mie with a low value of $n$ suggested by effective medium approximations to try to account for voids within the particles. When the values of $n$ and $k$ are known, using Mie with these values in the retrieval yields better lower limits than using Fraunhofer, as shown by Figure 7 and Figure 8.

It is worth noting that the minimum in the retrieval MSE $n$ - $k$ contour (e.g. Figure 9c) is not far from a relative minimum in the phase function residuals $n-k$ contour (Figure 9d), albeit they are generally different in all scenarios. Changing the values of $k$ and $n$ to some effective values in the retrieval model may help to get better agreement with the original $S(\log r)$, but this does not necessarily mean that the norm of the fit residuals of the average phase function is also at a minimum in the $n-k$ surface. In addition, the best retrieval of the size distribution is not obtained for a refractive index that brings the shape of the Mie $Q_{\text {sca }}(r)$ curve closer to that of the irregular particle, i.e. by changing $m$ in the retrieval in such a way that the peak efficiencies match and the Mie oscillations are smoothed.. This is because the differences between the forward and inversion scattering model phase functions $P_{11}(x, \theta)$ also reflect in the retrieved size distribution (Eq. 7). In fact, there are significant differences between the angular dependence of $P_{11}(x, \theta)$ for agglomerated debris and spheres, including the amplitude of oscillations and the Q-space slopes $[55,56]$. We do not seek to investigate the details of how the compensation of these differences for each $x$ governs the trends observed in Figure 8 and Figure 9. For the purpose of this paper, the scattering efficiency diagnostic gives sufficient insight into the origin of the submicron artefacts. 


\subsubsection{Varying particle morphology}

In principle, it could be expected that for particles with higher packing density than agglomerated debris and generated with a similar procedure, the behaviour of the phase function would be closer to that of spheres and perhaps better in line with using Mie theory with an effective medium approximation.
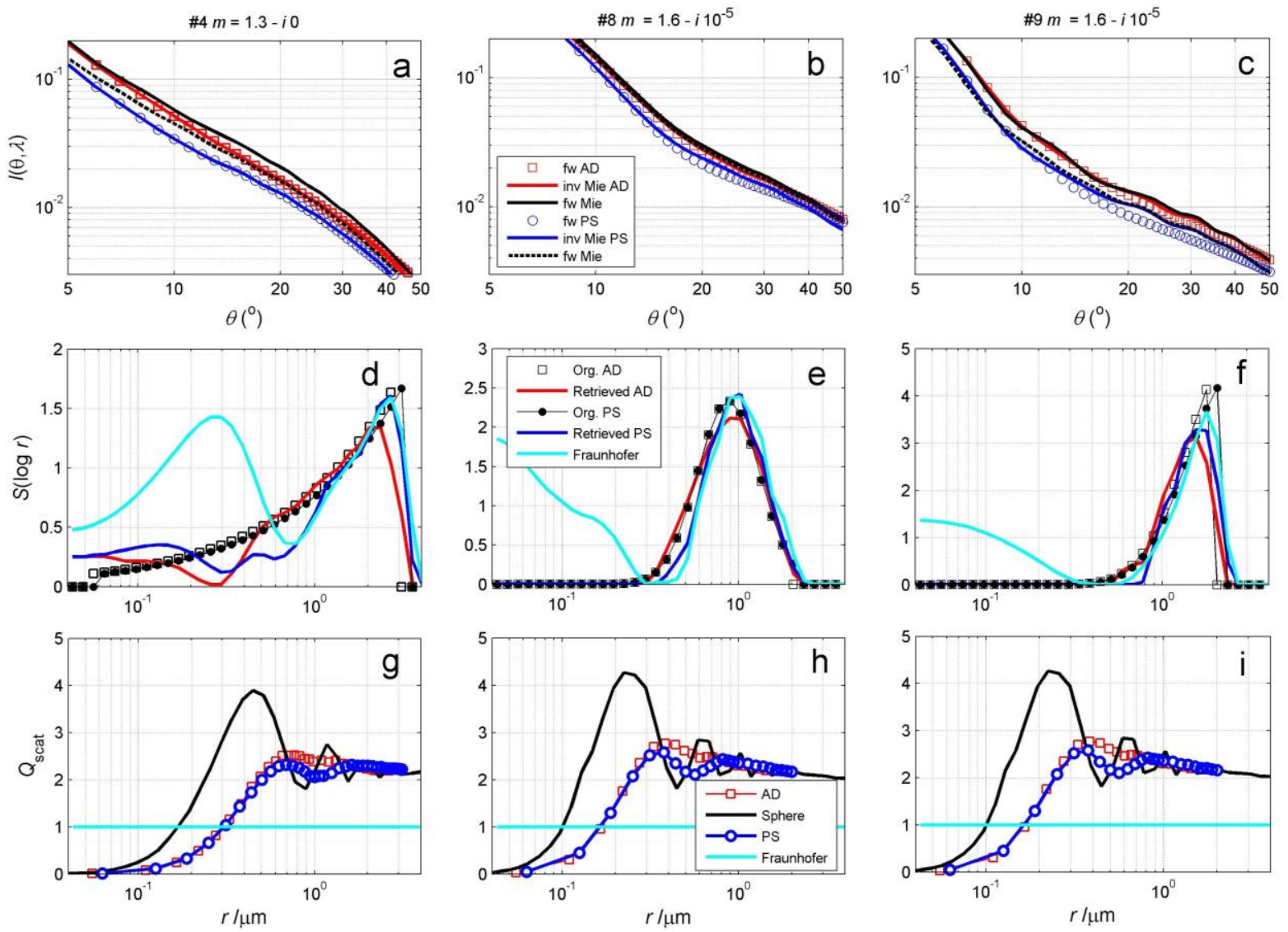

Figure 10. Comparison of retrievals of scenarios \#4, \#8 and \#9 for agglomerated debris (red) and pocked spheres (blue) at 442 $\mathrm{nm}$. Top panel row: average phase functions: forward DDA model for agglomerated debris (fw AD, red squares) and pocked spheres (fw PS, blue circles), respective Mie inversion fits (red and blue lines) and Mie forward model for spheres (size of AD: black line; size of PS: dashed black line). Middle panel row: retrieved $S(\log r)$ for agglomerated debris (red lines), pocked spheres (blue lines Mie, cyan Fraunhofer) and the respective original $S(\log r)$ distributions (black squares for agglomerated debris and black circles for pocked spheres). Bottom panel row: scattering efficiencies for agglomerated debris (red squares), pocked spheres (blue circles) and spheres (black lines). The size corresponds to the radius of the projected area-equivalent sphere.

Pocked spheres are model particles with agglomerated morphology, but with some residual spherical surfaces and with higher average packing density than agglomerated debris [47]. Thus, in principle they may be regarded as slightly less irregular than agglomerated debris. We have performed calculations for three different scenarios (\#4, \#8 and \#9) to investigate the effect of particle morphology on the LLS retrievals. Figure10 shows that the scattering efficiencies of agglomerated debris (AD) and pocked spheres (PS) are similar. For the same circumscribing sphere, pocked spheres have slightly larger cross sections, and therefore slightly larger radii, which results in narrower phase functions, lower at side scattering angles (Figure10, first panel row). Note that the inversion detects the larger size of pocked spheres (scenarios \#4 and \#9). For pocked spheres, $Q_{\text {sca }}$ shows somewhat more structure and the peak is slightly lower. The forward-modelled average phase functions for spheres lie above the corresponding 
phase functions for pocked spheres and agglomerated debris for the three scenarios, and for agglomerated debris the difference with spheres is smaller. Despite the small difference between the scattering efficiencies, the retrieved distributions for pocked spheres show wider slumps than for agglomerated debris. Contrary to what could be expected, the retrieved size distributions for pocked spheres are worse than for agglomerated debris.

\section{Discussion}

The LLS method works accurately for spherical particles of any arbitrary complex refractive index when the model employed by the inversion algorithm is Mie and the refractive index is well known. Under these circumstances, the upper size limit is determined by how close to $\theta=0$ is the first observation of the phase function. Similarly, the lower size limit is first determined by the upper limit the scattering angle range, with increased sensitivity towards smaller particles if larger scattering angles are available. Ultimately, the lower limit is determined by the Rayleigh regime boundary and is dependent on the wavelength employed by the analyser. Some commercial particle sizers appear to claim sensitivity down to $20 \mathrm{~nm}$ [20], which is clearly unrealistic as show by this work and previous studies [34], at least for instruments only based on the angular dependence of scattered intensity[39].

The results for spheres are sensitive to error in the refractive index used with the Mie inversion model if the true distribution includes a significant concentration of particles with sizes below the size where the maximum of the scattering efficiency of spheres occurs, e.g. $<1.5 \mu \mathrm{m}$ for $n=1.1$, and below for larger $n$. Deviations with respect to the original distribution are large when the true value of $k$ is larger than $\sim 0.05$, and the guessed value used in the inversion is smaller than this value, or vice versa. A similar boundary for $n$ can be tentatively placed at $n \sim 1.5$, although the sensitivity to small changes in $n$ when the true $n$ is less than 1.5 is much larger, and this is important when the dispersion medium is not air. All these deviations can be understood by inspecting the difference between the scattering efficiency of the inversion model and of the forward model, considering the range of particles that are encompassed by the original distribution. The limit value of $k \sim 0.05$ is in line with the $k x \sim 1$ criterion developed in the context of $\mathrm{Q}$ space analysis [57]. The $k x \sim 1$ criterion indicates when the imaginary part $k$ affects the scattering by a sphere. For example, in Figure 4, for $x=15.7(r=1.58 \mu \mathrm{m}$ at $\lambda=633$ $\mathrm{nm}), k x=0.8$.

If Fraunhofer is used instead of Mie as retrieval model, the lower size limit depends on the true refractive index of the spherical particles, being almost as good as Mie if the particles are very absorbing and becoming poorer as $n$ and $k$ decrease. Using the Fraunhofer approximation is similar to using Mie with high values of $n$ and $k$. The limit of applicability of the Fraunhofer method for spheres is conservatively placed at $\sim 3 \mu \mathrm{m}(\lambda=633 \mathrm{~nm})$ to consider the whole domain of relative refractive indices that can be found in real dust samples $(n>1, k \geq 0)$. This is significantly lower than the conservative theoretical lower limits usually found in the literature [19,29], and in agreement with empirical characterizations 
[39]. This limit would be slightly lower $(\sim 2 \mu \mathrm{m})$ if a blue source was used instead of the typical, He-Ne laser in the complete range of scattering angles. It is worth noting that Fraunhofer is usually said to be valid for small scattering angles (mainly because of the mismatch of the first secondary scattering lobe), while in practice a scattering angle range as wide as $0^{\circ} \leq \theta \leq 30^{\circ}$ can be used.

The size distributions retrieved for different types of irregular particles show artefacts that depend on the specific deviations of their phase functions with respect to those of spheres. As shown by the comparison between agglomerated debris and pocked spheres, these deviations are difficult to predict, since they do not depend in a straightforward manner on parameters such as packing density, even if the particle model precursors have spherical shape. Spheres are, in fact, not representative of compact particles, but only of themselves: ideal resonators with perfectly smooth surface. They are far from being a generally representative shape. The main reasons for using them as reference is that their size is defined by a single parameter (radius) and their scattering matrix can be exactly calculated using Mie theory with minimal computational effort. They are a useful model in particle sizing as far as shape effects do not become equal in magnitude to the size and refractive index dependences of the phase function. In fact, the LLS inversion method yields good retrievals even in the high end of the submicron range if the refractive index of the constituent material is known. It is worth highlighting that the behaviour relative to spheres described above is specific of agglomerated debris, and that the artefacts appearing in size distributions retrieved for other types of model particles may show different dependence on the refractive index determined by a different angular and size dependence of $P_{11}$.

It is not too surprising that effective medium approximations fail in providing better size distribution retrievals for agglomerated debris. For hollow spheres and porous pseudo-spheres with Rayleigh-sized inclusions, Mie theory combined with effective medium estimations of the refractive index provides good approximations to average phase functions and scattering efficiencies [58-60]. The manifestation of porosity $(1-\rho)$ is an effective refractive index (lower $n$ and lower $k$ ), which implies a shift of the peak $Q_{\text {sca }}$ towards larger sizes and an increase of the peak value itself [61]. The integral scattering properties of random gaussian spheres [62] are strongly influenced by their irregularity (characterized by the relative standard deviation $\sigma$ ), which smooths the oscillations in the resonance regime and reduces the peak $Q_{\text {scat }}$ [12]. The effect of increasing irregularity is qualitatively similar to decreasing $n$ and increasing $k$ for spheres. By analogy, the combined effect of porosity and irregularity in agglomerated debris and pocked spheres could be expected to be an effective reduction of the real part of the refractive index, and some kind of balance between a porosity-related effectively reduced $k$ and irregularityrelated effectively increased $k$. However, voids within the agglomerated debris and pocked spheres are larger than Rayleigh sizes, and therefore application of effective medium approximations as a way of emulating the effect of irregularity and porosity is dubious. Moreover, in order to maintain the same porosity in small and larger particles, the effective refractive index should be size-dependent (more voids are required in small spheres in order to simulate the same porosity than in large ones). 
The consequences of the present study could be far reaching. Scattering matrix elements measured in conjunction with LLS-retrieved size distributions (e.g. from the Amsterdam-Granada database [17]) have been used in the past to tune atmospheric aerosol retrieval algorithms. The AERONET retrieval method [16, 63] is based on a similar principle than the inversion method employed in this work, using mixtures of spheroids rather than spheres. This inversion method was trained on a cloud of small feldspar particles from scattering matrix laboratory measurements (available at [17]), to retrieve its size distribution and compare it to the distribution reported for this sample using LLS sizing with the Fraunhofer approximation [16, 64]. The LLS-derived distribution includes a significant number of submicrometric particles. Although the presence of a fraction of small particles can be real, the authors of this study noted that the Fraunhofer method is not suited for sizing submicrometric particles. This is confirmed by our study, which shows that the Fraunhofer approximation produces spuriously enhanced abundances of submicron particles for low $k$ materials, while, for micron-sized irregular particles it yields valid results. Thus, the AERONET inversion method can be considered to be well trained for irregular particles with sizes down to $1 \mu \mathrm{m}$. Below, it is possible that spheroids have similar problems than spheres in matching the shape-dependent features of the scattering properties of irregular particles. A hint of this is that they are not able to reproduce simultaneously the scattering matrix elements of feldspar at two wavelengths ( $\lambda=441$ and $\lambda=633 \mathrm{~nm}$ ) with the same size distribution. Thus, it cannot be entirely ruled out that extra modes and slumps appearing in some of the retrieved distributions are actually resulting from such a mismatch. Agglomerated debris do a better job in reproducing the scattering matrix elements of the same feldspar sample at the two wavelengths simultaneously in forward modelling [13], which suggests that inversion algorithms need to incorporate more elaborated models of irregular particles. This should also enable a more systematic implementation of the inversion of polarisation measurements by LLS particle sizers.

\section{Summary and conclusions}

In this work we have revisited the problem of sizing particles by inversion of an observed phase function at the core of the LLS sizing technique. This method is widely used in different scientific contexts, but it appears that there are several misconceptions about the meaning of its results and its range of applicability. We have performed a systematic analysis of the method for spherical particles using typical wavelengths and a range of common size distributions, which constrains the intrinsic applicability method using the Mie model with the correct refractive index to $r>0.1 \mu \mathrm{m}$ ( $r=$ radius of the projected area equivalent sphere), i.e. to the Mie scattering regime and above. Claims of sensitivity below this limit must be regarded with scepticism if additional supporting techniques (e.g. polarimetry) or aprioristic information (extrapolation) are not considered or insufficiently explained. Fraunhofer approximates a particular case of the Mie scattering model, that can be applied for $r>0.5 \mu \mathrm{m}$ for very absorbing particles and generally for $r>3 \mu \mathrm{m}(\lambda=633 \mathrm{~nm})$ if the refractive index is unknown. These limits would be reduced by a factor of $\sim 0.7$ if the measurements were carried out with a blue light 
source. Poor guesses of the refractive index manifest strongly when the estimated $n$ or $k$ are at the other side of the limits given by $n \sim 1.5$ and $k \sim 0.05$ with respect to the true index. Making extreme errors in the estimation of a refractive index is unlikely, but simulating them and visualizing the mismatch between the scattering efficiency of the forward model and the inversion model helps in understanding why the results of the LLS method for submicron irregular particles may be inaccurate. Note however that if the dispersion medium is water, a mid-range refractive index typical of e.g. silicates corresponds to a relative refractive index closer to the lower end of the range, where the sensitivity to the value of $n$ used in the retrieval is higher.

When using Mie with the same relative refractive index than the constituent material of the irregular particles to retrieve their size distribution, artefacts appear, which are related to the mismatch between the true and the Mie model scattering efficiency curves and normalized phase functions for single size bins. The onset of the artefacts depends on the real part of the relative complex refractive index and can be as low as $r \sim 0.3 \mu \mathrm{m}$ for $n=1.7(\lambda=442 \mathrm{~nm})$. Because dispersion media other than air are often used by LLS particle sizers, the lower limit of validity of the retrieved size distribution depends on the refractive index of the medium. Thus, for $n_{\mathrm{p}}=1.7$ and $n_{\mathrm{m}}=1.333$ (water), $n=1.27$, and the lower limit is placed at $r \sim 0.5 \mu \mathrm{m}$ instead. The shape of these artefacts depends on the value of $k$ and on the abundance of particles with sizes comprised within the size range where the mismatch occurs. The upper limit of the range where artefacts appear is placed conservatively at $r \sim 1 \mu \mathrm{m}(\lambda=442 \mathrm{~nm})$. This accounts for the worse results for pocked spheres than for agglomerated debris, as well as the poorer lower limit for low values of $n$. Effective refractive index approximations do not help in obtaining better results (i.e. an improved lower limit) - only for high absorption these approximations give some qualitative information. Owing to the smoothing effect of irregularity on $Q_{\text {sca }}$, the limit of Fraunhofer is lowered to $r \sim 1 \mu \mathrm{m}(\lambda=442 \mathrm{~nm})$ even for transparent particles. For samples with unknown $n$, Fraunhofer may even give a better lower limit for the retrieved distribution than an underestimation of $n$. Note however that Fraunhofer cannot be generally trusted for $r<1 \mu \mathrm{m}(\lambda=442 \mathrm{~nm})$, while Mie extends the validity almost to the lower limit for spheres if $n$ is high. If $n$ is uncertain, it is safer to assume values at the upper end of the uncertainty range when using the Mie model in the retrieval.

It appears unfortunately that a large uncertainty exists in the LLS method for aggregate irregular particles for $r<1 \mu \mathrm{m}$. The model particles used as illustrating examples in this work are extremely irregular and porous, and therefore we may be looking at worst case scenarios. Usually laboratory mineral and cosmic dust analogue samples present smoother surfaces and less porosity [42], which should make the mismatch in scattering efficiency less severe. Inspection of particle structure by obtaining scanning electron microscopy images of the samples analysed with the LLS technique, and a thorough analysis of the dependence of the retrieved size distribution on the assumed refractive index, should guide in deciding about their validity in the submicron range. 


\section{Acknowledgements}

This work has been funded by the Spanish Ministry of Science through the Instituto de Astrofísica de Andalucía-CSIC, Centro de Excelencia Severo Ochoa under grant SEV-2017-0709.

\section{References}

[1] Kolokolova L, Hough J, Levasseur-Regourd AC. Polarimetry of Stars and Planetary Systems. Cambridge, UK: Cambridge University Press; 2015.

[2] Moreno F, Guirado D, Muñoz O, Bertini I, Tubiana C, Güttler C, et al. Models of Rosetta/OSIRIS 67P Dust Coma Phase Function. The Astronomical Journal. 2018;156:237. doi:10.3847/15383881/aae526

[3] Zubko E, Videen G, Hines DC, Shkuratov Y. The positive-polarization of cometary comae. Plan Space Sci. 2016;123:63-76. doi:10.1016/j.pss.2015.09.020

[4] Kolokolova L, Das HS, Dubovik O, Lapyonok T, Yang P. Polarization of cosmic dust simulated with the rough spheroid model. Plan Space Sci. 2015;116:30-8. doi:10.1016/j.pss.2015.03.006

[5] Lasue J, Levasseur-Regourd AC, Hadamcik E, Alcouffe G. Cometary dust properties retrieved from polarization observations: Application to C/1995 O1 Hale-Bopp and 1P/Halley. Icarus. 2009;199:12944. doi:10.1016/j.icarus.2008.09.008

[6] Bertini I, Thomas N, Barbieri C. Modeling of the light scattering properties of cometary dust using fractal aggregates. A\&A. 2007;461:351-64. doi:10.1051/0004-6361:20065461

[7] Mishchenko MI, Hovenier JW, Travis LD. Light scattering by non-spherical particles. San Diego, USA: Academic Press; 2000.

[8] Dlugach JM, Ivanova OV, Mishchenko MI, Afanasiev VL. Retrieval of microphysical characteristics of particles in atmospheres of distant comets from ground-based polarimetry. J Quant Spectrosc Radiat Transfer. 2018;205:80-90. doi:10.1016/j.jqsrt.2017.10.002

[9] Hadamcik E, Lasue J, Levasseur-Regourd AC, Renard JB. Analogues of interplanetary dust particles to interpret the zodiacal light polarization. Plan Space Sci. 2018. doi:10.1016/j.pss.2018.04.022

[10] Muñoz O, Moreno F, Vargas-Martín F, Guirado D, Escobar-Cerezo J, Min M, et al. Experimental Phase Functions of Millimeter-sized Cosmic Dust Grains. Astrophys J. 2017;846:85. doi:10.3847/15384357/aa7ff2

[11] Frattin E, Muñoz O, Moreno F, Nava J, Escobar-Cerezo J, Gomez Martin JC, et al. Experimental phase function and degree of linear polarization of cometary dust analogues. Monthly Notices of the Royal Astronomical Society. 2019;484:2198-211. doi:10.1093/mnras/stz129 
[12] Liu J, Yang P, Muinonen K. Dust-aerosol optical modeling with Gaussian spheres: Combined invariant-imbedding T-matrix and geometric-optics approach. J Quant Spectrosc Radiat Transfer. 2015;161:136-44. doi:10.1016/j.jqsrt.2015.04.003

[13] Zubko E, Muinonen K, Muñoz O, Nousiainen T, Shkuratov Y, Sun W, et al. Light scattering by feldspar particles: Comparison of model agglomerate debris particles with laboratory samples. J Quant Spectrosc Radiat Transfer. 2013;131:175-87. doi:10.1016/j.jqsrt.2013.01.017

[14] Lindqvist H, Nousiainen T, Zubko E, Muñoz O. Optical modeling of vesicular volcanic ash particles. J Quant Spectrosc Radiat Transfer. 2011;112:1871-80. doi:10.1016/j.jqsrt.2011.01.032

[15] Penttilä A, Lumme K, Worms JC, Hadamcik E, Renard JB, Levasseur-Regourd AC. Theoretical analysis of the particle properties and polarization measurements made in microgravity. J Quant Spectrosc Radiat Transfer. 2003;79-80:1043-9. doi:10.1016/S0022-4073(02)00338-2

[16] Dubovik O, Sinyuk A, Lapyonok T, Holben BN, Mishchenko M, Yang P, et al. Application of spheroid models to account for aerosol particle nonsphericity in remote sensing of desert dust. Journal of Geophysical Research: Atmospheres. 2006;111. doi:10.1029/2005jd006619

[17] Muñoz O, Moreno F, Guirado D, Dabrowska DD, Volten H, Hovenier JW. The AmsterdamGranada Light Scattering Database. J Quant Spectrosc Radiat Transfer. 2012;113:565-74. doi:10.1016/j.jqsrt.2012.01.014

[18] Muñoz O, Moreno F, Guirado D, Ramos JL, López A, Girela F, et al. Experimental determination of scattering matrices of dust particles at visible wavelengths: The IAA light scattering apparatus. J Quant Spectrosc Radiat Transfer. 2010;111:187-96. doi:10.1016/j.jqsrt.2009.06.011

[19] ISO. ISO 13320-1 Particle Size Analysis - Guidance on laser diffraction methods. 2009.

[20] Rawle A. The importance of particle sizing to the coatings industry Part 1: Particle size measurement. Adv Col Sci Tech. 2002;5:1-12.

[21] Vlachos N, Chang ITH. Graphical and statistical comparison of various size distribution measurement systems using metal powders of a range of sizes and shapes. Powder Metall. 2011;54:497506. doi:10.1179/003258910x12707304455022

[22] Etzler FM, Deanne R. Particle size analysis: A comparison of various methods ii. Particle \& Particle Systems Characterization. 1997;14:278-82. doi:10.1002/ppsc.19970140604

[23] Kaye BH, Alliet D, Switzer L, Turbitt-Daoust C. The Effect of Shape on Intermethod Correlation of Techniques for Characterizing the Size Distribution of Powder. Part 1: Correlating the Size Distribution Measured by Sieving, Image Analysis, and Diffractometer Methods. Particle \& Particle Systems Characterization. 1997;14:219-24. doi:10.1002/ppsc.199700048 
[24] Neumann AM, Kramer HJM. A Comparative Study of Various Size Distribution. Particle \& Particle Systems Characterization. 2002;19:17-27. doi:10.1002/1521-4117(200204)19:1<17::aidppsc17>3.0.co;2-1

[25] Hansen JE, Travis LD. Light scattering in planetary atmospheres. Space Sci Rev. 1974;16:527-610. doi:10.1007/bf00168069

[26] Escobar-Cerezo J, Muñoz O, Moreno F, Guirado D, Gómez Martín JC, Goguen JD, et al. An Experimental Scattering Matrix for Lunar Regolith Simulant JSC-1A at Visible Wavelengths. Astrophys J Supp Ser. 2018;235:19. doi:10.3847/1538-4365/aaa6cc

[27] Shkuratov Y. On the origin of the opposition effect and negative polarization for cosmic bodies with solid surface. Astron Circ. 1985;1400:3-6.

[28] Muinonen K. Electromagnetic scattering by two interacting dipoles. URSIEM theory symposium1989. p. 428-30.

[29] van de Hulst HC. Light Scattering by Small Particles. New York: Dover; 1957.

[30] Hovenier JW, van der Mee CVM, Domke H. Transfer of Polarized Light in Planetary Atmospheres Basic Concepts and Practical Methods. Dordrecht: Kluwer Springer Netherlands; 2004.

[31] Mishchenko MI, Travis LD, Lacis AA. Scattering, absorption and emission of light by small particles. Cambridge2002.

[32] Dubovik O, King MD. A flexible inversion algorithm for retrieval of aerosol optical properties from Sun and sky radiance measurements. J Geophys Res [Atmos]. 2000;105:20673-96. doi:10.1029/2000jd900282

[33] Andrews S, Nover D, Schladow SG. Using laser diffraction data to obtain accurate particle size distributions: the role of particle composition. Limnology and Oceanography: Methods. 2010;8:50726. doi:10.4319/lom.2010.8.507

[34] Veihelmann B, Konert M, van der Zande WJ. Size distribution of mineral aerosol: using lightscattering models in laser particle sizing. Appl Opt. 2006;45:6022-9. doi:10.1364/ao.45.006022

[35] Tikhonov AN, Arsenin VIA. Solutions of ill-posed problems: Winston; 1977.

[36] Hansen PC. Regularization Tools version 4.0 for Matlab 7.3. Numer Algorithms. 2007;46:189-94. doi:10.1007/s11075-007-9136-9

[37] King MD. Sensitivity of Constrained Linear Inversions to the Selection of the Lagrange Multiplier. J Atmos Sci. 1982;39:1356-69. doi:10.1175/1520-0469(1982)039<1356:soclit>2.0.co;2 
[38] Boxman A, Merkus HG, Verheijen PJT, Scarlett B. Deconvolution of light-scattering patterns by observing intensity fluctuations. Appl Opt. 1991;30:4818-23. doi:10.1364/ao.30.004818

[39] Keck CM, Müller RH. Size analysis of submicron particles by laser diffractometry- $90 \%$ of the published measurements are false. Int J Pharm. 2008;355:150-63. doi:10.1016/j.ijpharm.2007.12.004

[40] Heinson WR, Chakrabarti A, Sorensen CM. Crossover from spherical particle Mie scattering to circular aperture diffraction. J Opt Soc Am A. 2014;31:2362-4. doi:10.1364/josaa.31.002362

[41] Mishchenko MI. Lorenz-Mie FORTRAN Programs for Computing Far-Field Electromagnetic Scattering by Monodisperse and Polydisperse Spherical Particles. https://www.giss.nasa.gov/staff/mmishchenko/Lorenz-Mie.html. 2002.

[42] CODULAB-IAA-CSIC. The Amsterdam-Granada scattering database. https://www.iaa.csic.es/scattering/. 2019.

[43] Zubko E, Shkuratov Y, Kiselev NN, Videen G. DDA simulations of light scattering by small irregular particles with various structure. J Quant Spectrosc Radiat Transfer. 2006;101:416-34. doi:10.1016/j.jqsrt.2006.02.055

[44] Zubko ES. Light scattering by irregularly shaped particles with sizes comparable to the wavelength. In: Kokhanovsky AA, editor. Light Scattering Reviews, Vol 6: Light Scattering and Remote Sensing of Atmosphere and Surface. Berlin, Heidelberg: Springer Berlin Heidelberg; 2012. p. 39-74.

[45] Zubko E. Modeling light scattering by forsterite particles. Opt Lett. 2015;40:1204-7. doi:10.1364/ol.40.001204

[46] Kelly RN, Kazanjian J. Commerical reference shape standards use in the study of particle shape effect on laser diffraction particle size analysis. AAPS PharmSciTech. 2006;7:E126-E37. doi: $10.1208 / \mathrm{pt} 070249$

[47] Zubko E, Shkuratov Y, Videen G. Effect of morphology on light scattering by agglomerates. J Quant Spectrosc Radiat Transfer. 2015;150:42-54. doi:10.1016/j.jqsrt.2014.06.023

[48] Nousiainen T, Zubko E, Lindqvist H, Kahnert M, Tyynelä J. Comparison of scattering by different nonspherical, wavelength-scale particles. J Quant Spectrosc Radiat Transfer. 2012;113:2391-405. doi:10.1016/j.jqsrt.2012.03.032

[49] Twomey S. Comparison of constrained linear inversion and an iterative nonlinear algorithm applied to the indirect estimation of particle size distributions. Journal of Computational Physics. 1975;18:188200. doi:10.1016/0021-9991(75)90028-5

[50] Igushi T, Yoshida H. Investigation of low-angle laser light scattering patterns using the modified Twomey iterative method for particle sizing. Rev Sci Instrum. 2011;82:015111. doi:10.1063/1.3520136 
[51] Sequoia. Laser Diffraction 201 - LISST. https://www.sequoiasci.com/article/laser-diffraction-201lisst/. 2011.

[52] LPC2E-CNRS. PROGRA2 Database. http://www.icare.univ-lille1.fr/progra2/database/index.html. 2015.

[53] Hansen PC. Regtools. https://es.mathworks.com/matlabcentral/fileexchange/52-regtools. 2003.

[54] Lawson CL, Hanson RJ. Solving Least Squares Problems. Englewood Cliffs, NJ: Prentice-Hall; 1974.

[55] Videen G, Zubko E, Sun W, Shkuratov Y, Yuffa A. Mixing rules and morphology dependence of the scatterer. J Quant Spectrosc Radiat Transfer. 2015;150:68-75. doi:10.1016/j.jqsrt.2014.07.022

[56] Sorensen CM, Zubko E, Heinson WR, Chakrabarti A. Q-space analysis of scattering by small irregular particles. J Quant Spectrosc Radiat Transfer. 2014;133:99-105. doi:https://doi.org/10.1016/j.jqsrt.2013.07.020

[57] Wang G, Chakrabarti A, Sorensen CM. Effect of the imaginary part of the refractive index on light scattering by spheres. J Opt Soc Am A. 2015;32:1231-5. doi:10.1364/josaa.32.001231

[58] Lumme K, Rahola J. Light Scattering by Porous Dust Particles in the Discrete-Dipole Approximation. Astrophys J. 1994;425:653. doi:10.1086/174012

[59] Voshchinnikov NV, Il'in VB, Henning T. Modelling the optical properties of composite and porous interstellar grains. A\&A. 2005;429:371-81. doi:10.1051/0004-6361:200400081

[60] Voshchinnikov NV, Videen G, Henning T. Effective medium theories for irregular fluffy structures: aggregation of small particles. Appl Opt. 2007;46:4065-72. doi:10.1364/ao.46.004065

[61] Vilaplana R, Luna R, Guirado D. The shape influence on the overall single scattering properties of a sample in random orientation. J Quant Spectrosc Radiat Transfer. 2011;112:1838-47. doi:10.1016/j.jqsrt.2011.01.006

[62] Muinonen K, Nousiainen T, Fast P, Lumme K, Peltoniemi JI. Light scattering by Gaussian random particles: Ray optics approximation. J Quant Spectrosc Radiat Transfer. 1996;55:577-601. doi:10.1016/0022-4073(96)00003-9

[63] Dubovik O, Holben BN, Lapyonok T, Sinyuk A, Mishchenko MI, Yang P, et al. Non-spherical aerosol retrieval method employing light scattering by spheroids. Geophys Res Lett. 2002;29:54-1--4. doi:10.1029/2001g1014506

[64] Volten H, Muñoz O, Rol E, de Haan JF, Vassen W, Hovenier JW, et al. Scattering matrices of mineral aerosol particles at $441.6 \mathrm{~nm}$ and $632.8 \mathrm{~nm}$. Journal of Geophysical Research: Atmospheres. 2001;106:17375-401. doi:10.1029/2001jd900068 
